\title{
Pacific
}

Journal of

Mathematics

\section{A TOPOLOGICAL CONSTRUCTION FOR ALL TWO-ROW SPRINGER VARIETIES}

\author{
HEATHER M. RUSSELL
}




\title{
A TOPOLOGICAL CONSTRUCTION FOR ALL TWO-ROW SPRINGER VARIETIES
}

\author{
HEATHER M. RusSELL
}

\begin{abstract}
Springer varieties appear in both geometric representation theory and knot theory. Motivated by knot theory and categorification, Khovanov provides a topological construction of $(m, m)$ Springer varieties. Here we extend his construction to all two-row Springer varieties. Using the combinatorial and diagrammatic properties of this construction we provide a particularly useful homology basis and construct the Springer representation using this basis. We also provide a skein-theoretic formulation of the representation in this case.
\end{abstract}

\section{Introduction}

Springer varieties (or Springer fibers) are certain subvarieties of the variety of full flags in $\mathbb{C}^{n}$. Given a partition $\lambda$ of the number $n$ the Springer variety $\mathscr{Y}_{\lambda}$ is the collection of full flags in $\mathbb{C}^{n}$ fixed by a nilpotent linear operator with Jordan blocks given by $\lambda$. Springer varieties were first introduced by Springer who constructed irreducible representations of the symmetric group on their top nonzero cohomology classes [Springer 1976]. This remarkable construction has motivated the study of these varieties by geometric representation theorists; see, for example, [Fung 2003; Hotta 1981; Kazhdan and Lusztig 1980].

This representation of the symmetric group, which has come to be known as the Springer representation, appears in the literature in a variety of forms. This includes Springer's original representation [1976] on the cohomology of Springer varieties, the tensor product of Springer's representation with the sign representation [Lusztig 1981; Slodowy 1980; Borho and MacPherson 1981], and a representation on homology which is isomorphic to Springer's cohomology representation [Kazhdan and Lusztig 1980]. Many of these equivalences are proven in [Hotta 1981].

A significant part of this paper was written in Spring 2010 while attending the Homology Theories of Knots and Links program at the Mathematical Sciences Research Institute. Thank you to MSRI for this opportunity. The author was also partially supported by NSF VIGRE grant DMS 0739382. MSC2000: 20C30, 55N45, 57M25, 57M60.

Keywords: two-row Springer variety, Springer representation, noncrossing matchings, tangle homology. 
Springer varieties have also begun to appear with increasing frequency in the literature on knot homologies and categorification. For $n=2 m$ Khovanov constructs a functor-valued invariant of tangles using an arc algebra $H^{m}$ with center isomorphic to the cohomology of the $(m, m)$ Springer variety [Khovanov 2002]. In proving this isomorphism he provides a topological construction of the $(m, m)$ Springer variety as a subspace of a product of spheres [Khovanov 2004]. Springer varieties also appear in Cautis-Kamnitzer knot homology via derived categories of coherent sheaves and in the Seidel-Smith link invariant from the symplectic geometry of nilpotent slices [Seidel and Smith 2006].

Stroppel gave a Lie-theoretic generalization [2005] of Khovanov's functor-valued invariant of tangles, and described diagrammatically [2009] the categories related to this construction and the top-degree cohomology of $(m, m)$-Springer varieties, using them to build the Springer representation. In this paper we consider homology rather than cohomology and all degrees rather than just the top degree.

The structure of Springer varieties is not well understood. In particular for general classes of Springer varieties the topology of individual components and the interaction of those components in not known. Finding topological models for Springer varieties has the potential to aid geometric representation theory as well as deepen our understanding of the connections between Springer varieties and knot theory.

In previous work we use Khovanov's topological construction to prove an isomorphism between the homology of the $(m, m)$ Springer variety and the Bar-Natan skein module of the solid torus with boundary web $2 m$ copies of the longitude [Russell 2009]. We also use this construction to give a completely explicit and combinatorial construction of Springer's representation on this class of Springer varieties [Russell and Tymoczko 2008].

Now consider $n$ not necessarily even. The first main result of this paper is an extension of Khovanov's construction of a topological model for $(m, m)$ Springer varieties to all Springer varieties $\mathscr{S}_{\lambda}$ where $\lambda$ is a two element partition of the number $n$. This construction can be found in Section 2. The proof that our construction is homeomorphic to the Springer variety follows the structure and approach of [Russell and Tymoczko 2008, Appendix].

We draw the reader's attention to the fact that the object we construct and the Springer variety are homeomorphic as topological spaces and not isomorphic as algebraic varieties. For our purposes, this is sufficient since we are interested in studying the homology of the Springer variety. Throughout the paper we consider singular homology with complex coefficients, and we refer to the homeomorphic image of the irreducible components of the variety as irreducible components.

In Section 3 we analyze the intersection of irreducible components of two-row Springer varieties and build an exact sequence on homology. In order to construct 
this sequence we generalize certain lemmas stated but not proven in [Khovanov 2004]. We prove these results in the Appendix.

In Section 4 we introduce diagrammatic generators for the homology of each component of a two-row Springer variety. We give three simple relations between the generators from different components within the same variety; these relations generalize those given in [Russell 2009]. We then use the exact sequence constructed in Section 3 to prove Theorem 4.3 which shows that these diagrammatic generators and relations describe the homology of the associated two-row Springer variety. We use these generators and relations to reduce to a basis.

Section 5 gives a completely diagrammatic construction of a symmetric group action on the homology of the $(n-k, k)$ Springer variety. As in [Russell and Tymoczko 2008], we prove it is isomorphic to the Springer representation by comparing it with the de Concini-Procesi construction of Springer representations [De Concini and Procesi 1981], making use of Garsia and Procesi's combinatorial analysis [1992] of de Concini and Procesi's work.

In response to a question of Stephan Wehrli we conclude Section 5 with the following theorem, which provides a skein-theoretic formulation of the Springer representation in the two-row case.

Theorem. Given a diagrammatic homology generator $M \in H_{*}\left(\mathscr{Y}_{n-k, k}\right)$ and $\sigma \in S_{n}$ glue a flattened braid corresponding to $\sigma$ to the bottom of $M$ forming $M^{\prime}$. Then the Springer action $\sigma \cdot M$ is equal to $s\left(M^{\prime}\right)$, where $s$ is defined as follows.

$$
\begin{aligned}
& s(X)=s(\vdots)+s(\vdots) \quad s(\vdots)=0 \\
& s\left(M^{\prime} \sqcup \bigcirc\right)=s\left(-2 M^{\prime}\right) \quad s\left(M^{\prime} \sqcup \bigcirc\right)=0
\end{aligned}
$$

\section{Extending Khovanov's topological construction}

For $n=2 m$ Khovanov constructs a topological space with cohomology isomorphic to that of the $(m, m)$ Springer variety and conjectures that the two are actually homeomorphic [Khovanov 2004, Conjecture 1]. The main ideas behind a proof of this fact can be found in [Cautis and Kamnitzer 2008], and detailed proofs are given in [Russell and Tymoczko 2008, Appendix] and independently in [Wehrli 2009]. In this section we generalize Khovanov's construction to all two-row Springer varieties. The proof that our construction is homeomorphic to the Springer variety follows that of [Russell and Tymoczko 2008, Appendix].

Let $n \geq 1$ be some positive integer. A complete flag in $\mathbb{C}^{n}$, denoted by $V_{\bullet}$, is a collection of nested subspaces

$$
V_{1} \subset V_{2} \subset \cdots \subset V_{n-1} \subset V_{n}
$$


such that the complex dimension of $V_{i}$ is $i$. The collection of all such objects is the variety we denote by $\mathscr{F}_{n}$. Partial flags have the same nesting property but are not required to have subsets in every intermediate dimension.

Let $\lambda=\left(\lambda_{1} \geq \lambda_{2} \geq \cdots \geq \lambda_{k}\right)$ be a partition of the number $n$. Let $\Gamma: \mathbb{C}^{n} \rightarrow \mathbb{C}^{n}$ be a nilpotent linear operator with Jordan blocks of sizes specified by the partition $\lambda$. Then we have the following definition.

Definition 2.1. The Springer variety associated to the partition $\lambda$ is

$$
\mathscr{S}_{\lambda}=\left\{V_{\bullet} \in \mathscr{F}_{n}: \Gamma V_{i} \subseteq V_{i} \text { for all } i\right\} .
$$

We focus on Springer varieties associated to two-element partitions which we call two-row Springer varieties. Note that every two-element partition of $n$ can be written as $(n-k, k)$ for some positive integer $0 \leq k \leq\left\lfloor\frac{n}{2}\right\rfloor$. We call $\mathscr{Y}_{n-k, k}$ the $(n-k, k)$ Springer variety.

A Young diagram for the partition $\lambda$ of the number $n$ is a top and left justified collection of boxes such that the $i$-th row has $\lambda_{i}$ boxes. A standard filling of this diagram is a filling of the boxes with the numbers 1 through $n$ such that each occurs exactly once and the rows and columns are strictly decreasing from left to right and top to bottom respectively.

The irreducible components of Springer varieties are indexed by standard Young tableaux [Spaltenstein 1976; Vargas 1979]. In the two-row case, the tableaux of shape $(n-k, k)$ are in one-to-one correspondence with certain noncrossing matchings which we will call noncrossing matchings of type $(n-k, k)$. This was noticed in [Stroppel and Webster 2010].

Definition 2.2. Consider $n$ vertices evenly spaced along a horizontal line. A noncrossing matching of type $(n-k, k)$ is a nonintersecting arrangement of $k$ arcs and $n-2 k$ rays incident on the $n$ vertices lying above the horizontal line. We assume that the rays are "infinitely high", so that arcs cannot cross rays. Here is an example of a noncrossing matching of type $(6,5)$ :

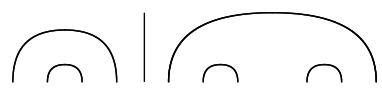

You can get a unique noncrossing matching of type $(n-k, k)$ from a standard Young tableau via the following procedure, illustrated in Figure 1. Start with the smallest number in the bottom row, draw an arc with right endpoint incident on that

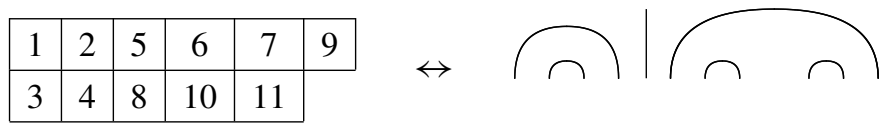

Figure 1. Going from a Young tableau to a noncrossing matching. 
vertex and left endpoint incident on the nearest unoccupied vertex. Repeat this for all remaining numbers in the bottom row, moving from left to right in the bottom row. Fill any remaining unoccupied positions with rays. One can easily define an inverse operation and see this is indeed a bijection. We generalize this procedure in the proof Lemma 4.7, so the reader can go there for more details.

Matchings will be denoted by lowercase letters. For clarity we will make these bold throughout. Let $B^{n-k, k}$ be the set of noncrossing matchings of type $(n-k, k)$. Given $\boldsymbol{a} \in B^{n-k, k}$ write $(i, j) \in \boldsymbol{a}$ if $\boldsymbol{a}$ has an arc connecting vertices $i$ and $j$. Write (i) $\in \boldsymbol{a}$ if $\boldsymbol{a}$ has a ray incident on the vertex $i$.

Let $N>2 n$ be a large fixed integer. Let $X: \mathbb{C}^{2 N} \rightarrow \mathbb{C}^{2 N}$ be a nilpotent linear operator with two Jordan blocks of size $N$. Let $\left\{e_{1}, \ldots, e_{N}, f_{1}, \ldots, f_{N}\right\}$ be an orthonormal basis for $\mathbb{C}^{2 N}$ with the property that $X e_{i}=e_{i-1}$ and $X f_{i}=f_{i-1}$. Here we define $e_{-1}=f_{-1}=0$.

Consider the following variety $Y_{n}$ of partial flags in $\mathbb{C}^{2 N}$.

$$
Y_{n}=\left\{V_{\bullet}=V_{1} \subset \cdots \subset V_{n}: \operatorname{dim}_{\mathbb{C}}\left(V_{i}\right)=i \text { and } X V_{i} \subseteq V_{i-1}\right\} .
$$

From now on flags will be written $\left(V_{1}, \ldots, V_{n}\right)$ to save space. For $0 \leq k \leq\left\lfloor\frac{n}{2}\right\rfloor$, let

$$
V_{n-k, k}=\left\langle e_{1}, \ldots, e_{n-k}, f_{1}, \ldots, f_{k}\right\rangle
$$

and define $Y_{n-k, k}=\left\{V_{\bullet} \in Y_{n}: V_{n}=V_{n-k, k}\right\}$. Since $Y_{n-k, k}$ is the set of all complete flags on $V_{n-k, k}$ fixed by $X$, it is diffeomorphic to $\mathscr{S}_{n-k, k}$.

For $1 \leq i \leq n$ define a subvariety of $Y_{n}$ by

$$
Z_{n}^{i}=\left\{V_{\bullet} \in Y_{n}: V_{i+1}=X^{-1} V_{i-1}\right\} .
$$

Define the map

$$
q: Z_{n}^{i} \rightarrow Y_{n-2}, \quad V_{\bullet}=\left(V_{1}, \ldots V_{n}\right) \mapsto V_{\bullet}^{\prime}=\left(V_{1}, \ldots, V_{i-1}, X V_{i+2}, \ldots, X V_{n}\right) .
$$

This map is a $\mathbb{P}^{1}$ bundle [Cautis and Kamnitzer 2008, page 5]. To see this, suppose that we have $V_{\bullet}^{\prime}=\left(V_{1}^{\prime}, \ldots, V_{n-2}^{\prime}\right) \in Y_{n-2}$ and are considering possible choices of $V_{\bullet} \in Z_{n}^{i}$ with $q\left(V_{\bullet}\right)=V_{\bullet}^{\prime}$. Then we must have $X V_{i+2}=V_{i}^{\prime}$ and $V_{i-1}=V_{i-1}^{\prime}$. Since $V_{i-1}^{\prime} \subset V_{i}^{\prime}$ and $V_{\bullet} \in Z_{n}^{i}$ we have a $\mathbb{P}^{1}$ worth of choices for $V_{i}$.

Let $p=(0,0,1)$ be the north pole of the standard unit two-sphere $S^{2}$ embedded in $\mathbb{R}^{3}$. Let $-p=(0,0,-1)$ be the south pole in $S^{2}$ where $-p$ is understood as the antipodal map.

Definition 2.3. Given $\boldsymbol{a} \in B^{n-k, k}$, define subspaces of $\left(S^{2}\right)^{n}$ by

$$
\begin{aligned}
& S_{\boldsymbol{a}, n-k, k}=\left\{\left(x_{1}, \ldots, x_{n}\right) \in\left(S^{2}\right)^{n}: x_{i}=x_{j} \text { if }(i, j) \in \boldsymbol{a} \text { and } x_{i}=(-1)^{i} p \text { if }(i) \in \boldsymbol{a}\right\}, \\
& S_{\boldsymbol{a}, n-k, k}^{\prime}=\left\{\left(x_{1}, \ldots, x_{n}\right) \in\left(S^{2}\right)^{n}: x_{i}=-x_{j} \text { if }(i, j) \in \boldsymbol{a} \text { and } x_{i}=p \text { if }(i) \in \boldsymbol{a}\right\} .
\end{aligned}
$$


Taking unions over all $\boldsymbol{a} \in B^{n-k, k}$ define

$$
X_{n-k, k}=\bigcup_{\boldsymbol{a}} S_{\boldsymbol{a}, n-k, k} \subset\left(S^{2}\right)^{n} \quad \text { and } \quad X_{n-k, k}^{\prime}=\bigcup_{\boldsymbol{a}} S_{\boldsymbol{a}, n-k, k}^{\prime} \subset\left(S^{2}\right)^{n} .
$$

The space $X_{n-k, k}$ is a generalization of Khovanov's construction for $n=2 m$ of the $(m, m)$ Springer variety [Khovanov 2004, page 4]. Indeed in the $(m, m)$ case matchings have no rays, so each component is built by identifying coordinates pairwise as prescribed by the arcs of the associated matching.

Using the basis $\left\{e_{1}, \ldots, e_{N}, f_{1}, \ldots, f_{N}\right\}$ for $\mathbb{C}^{2 N}$, write points in $\mathbb{P}^{2 N-1}$ in the form $\left\langle\sum a_{i} e_{i}+\sum b_{i} f_{i}\right\rangle$, for $a_{i}, b_{i} \in \mathbb{C}$. For $\mathbb{P}^{1}$ write $e_{1}=e$ and $f_{1}=f$. Define the map $C: \mathbb{P}^{2 N-1} \rightarrow \mathbb{P}^{1}$ by

$$
\left\langle\sum a_{i} e_{i}+\sum b_{i} f_{i}\right\rangle \mapsto\left\langle\left(\sum a_{i}\right) e+\left(\sum b_{i}\right) f\right\rangle .
$$

For each $V_{\bullet} \in Y_{n}$ define lines $L_{1}, \ldots, L_{n}$ by $V_{i}=V_{i-1} \oplus L_{i}$ and $L_{i} \perp V_{i-1}$.

Proposition 2.4 [Cautis and Kamnitzer 2008, Theorem 2.1]. The map

$$
\ell: Y_{n} \rightarrow\left(\mathbb{P}^{1}\right)^{n}
$$

defined by

$$
V_{\bullet} \mapsto\left(C\left(L_{1}\right), C\left(L_{2}\right), \ldots, C\left(L_{n}\right)\right)
$$

is a diffeomorphism. Furthermore the image of $Z_{n}^{i}$ under the diffeomorphism $\ell$ is exactly the elements in $\left(\mathbb{P}^{1}\right)^{n}$ satisfying $C\left(L_{i}\right)=-C\left(L_{i+1}\right)$.

Let $s: S^{2}-\{p\} \rightarrow \mathbb{C}$ be the stereographic projection, and let $\varphi: \mathbb{P}^{1}-\{\langle e\rangle\} \rightarrow \mathbb{C}$ be defined by $\varphi(\langle x e+y f\rangle)=x / y$. Let $\Phi: \mathbb{P}^{1} \rightarrow S^{2}$ be the diffeomorphism defined by

$$
\Phi(\langle x e+y f\rangle)= \begin{cases}s^{-1} \circ \varphi(\langle x e+y f\rangle) & \text { if }\langle x e+y f\rangle \neq\langle e\rangle, \\ (0,0,1) & \text { if }\langle x e+y f\rangle=\langle e\rangle .\end{cases}
$$

Define the diffeomorphism $\widetilde{\Phi}:\left(\mathbb{P}^{1}\right)^{n} \rightarrow\left(S^{2}\right)^{n}$ by

$$
\widetilde{\Phi}\left(x_{1}, \ldots, x_{n}\right)=\left(\Phi\left(x_{1}\right), \ldots, \Phi\left(x_{n}\right)\right) .
$$

Given $\boldsymbol{a} \in B^{n-k, k}$ define $C_{\boldsymbol{a}, n-k, k}$ to be the preimage $\ell^{-1} \circ \widetilde{\Phi}^{-1}\left(S_{\boldsymbol{a}, n-k, k}^{\prime}\right)$.

For $k>0$ let $\boldsymbol{a} \in B^{n-k, k}$ such that $(i, i+1) \in \boldsymbol{a}$, and let $\boldsymbol{a}^{\prime} \in B^{n-k-1, k-1}$ be the noncrossing matching obtained from $\boldsymbol{a}$ by erasing the $\operatorname{arc}(i, i+1)$. If $f$ : $\{1,2, \ldots, n\} \rightarrow\{1,2, \ldots, n-2\}$ is the map

$$
f(j)= \begin{cases}j & \text { if } j \leq i-1 \\ j-2 & \text { if } j \geq i+2\end{cases}
$$

then $\boldsymbol{a}^{\prime}$ is comprised of the set of arcs

$$
\left\{\left(f(j), f\left(j^{\prime}\right)\right):\left(j, j^{\prime}\right) \in \boldsymbol{a} \text { and }\left(j, j^{\prime}\right) \neq(i, i+1)\right\}
$$


and the set of rays $\{(f(j)):(j) \in \boldsymbol{a}\}$. Define the projection map $q^{\prime}: S_{\boldsymbol{a}, n-k, k}^{\prime} \rightarrow$ $S_{\boldsymbol{a}^{\prime}, n-k-1, k-1}^{\prime}$ by $q^{\prime}\left(x_{1}, \ldots, x_{n}\right)=\left(x_{1}, \ldots, \widehat{x_{i}}, \widehat{x_{i+1}}, \ldots, x_{n}\right)$ where $\widehat{x_{j}}$ omits the $j$-th coordinate.

Lemma 2.5. For noncrossing matchings $\boldsymbol{a}$ and $\boldsymbol{a}^{\prime}$ as above there is a commutative diagram

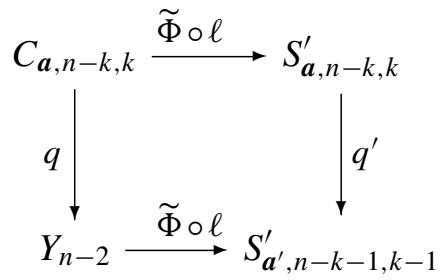

and the image $q\left(C_{\boldsymbol{a}, n-k, k}\right)$ is $C_{\boldsymbol{a}^{\prime}, n-k-1, k-1}$.

Proof. We provide a short proof here. For more details see [Russell and Tymoczko 2008, Lemma 5.2], which contains an identical argument.

Since the coordinates $x_{i}$ and $x_{i+1}$ in $S_{a, n-k, k}^{\prime}$ are antipodes, Proposition 2.4 allows us to conclude $C_{\boldsymbol{a}, n-k, k} \subseteq Z_{n}^{i}$. Let $V_{\bullet} \in Z_{n}^{i}$. By the definition of $Z_{n}^{i}$ we know that $X^{-1} V_{i-1}=V_{i+1}$ and thus ker $X \subseteq V_{i+1}$. It follows that $L_{j}$ is spanned by $\sum_{i \geq 2} a_{i} e_{i}+b_{i} f_{i}$ and $X L_{j} \perp X V_{j-1}$ for all $j \geq i+2$. This shows that if $V_{\bullet} \in C_{\boldsymbol{a}, n-k, k}$ then $(\widetilde{\Phi} \circ \ell)\left(q\left(V_{\bullet}\right)\right)=q^{\prime}\left((\widetilde{\Phi} \circ \ell)\left(V_{\bullet}\right)\right)$. By commutativity of the diagram, we conclude that $q\left(C_{\boldsymbol{a}, n-k, k}\right)$ is $C_{\boldsymbol{a}^{\prime}, n-k-1, k-1}$.

Lemma 2.6. The union $\bigcup_{\boldsymbol{a} \in B^{n-k, k}} C_{\boldsymbol{a}, n-k, k}$ is equal to $Y_{n-k, k}$. The $C_{\boldsymbol{a}, n-k, k}$ are the irreducible components of $Y_{n-k, k}$.

Proof. Consider the partition $(n, 0)$. The unique noncrossing matching $\boldsymbol{a} \in B^{n, 0}$ has $n$ rays and no arcs. Then $X_{n, 0}^{\prime}=S_{a, n, 0}^{\prime}=\left\{(p, \ldots, p) \in\left(S^{2}\right)^{n}\right\}$ and

$$
\widetilde{\Phi}^{-1}\left(S_{a, n, 0}^{\prime}\right)=\left\{(\langle e\rangle, \ldots,\langle e\rangle) \in\left(\mathbb{P}^{1}\right)^{n}\right\} .
$$

We want to find $V_{\bullet} \in Y_{n}$ such that $\left.\ell_{\left(V_{\bullet}\right.}\right)=(\langle e\rangle, \ldots,\langle e\rangle)$.

Since $C\left(L_{1}\right)=\langle e\rangle$ we must have $L_{1}=V_{1}=\left\langle e_{1}\right\rangle$. Inductively assume that $V_{i-1}=$ $\left\langle e_{1}, \ldots, e_{i-1}\right\rangle$. We must have $L_{i} \perp V_{i-1}$ and $X L_{i} \subset V_{i-1}$ so $L_{i}=\left\langle x_{i} e_{i}+y_{1} f_{1}\right\rangle$. Since $C\left(L_{i}\right)=\langle e\rangle$ this forces $y_{1}=0$. This shows

$$
\begin{aligned}
\left(\ell^{-1} \circ \widetilde{\Phi}^{-1}\right)\left(S_{\boldsymbol{a}, n, 0}^{\prime}\right) & =C_{\boldsymbol{a}, n, 0}=\left\{\left(\left\langle e_{1}\right\rangle,\left\langle e_{1}, e_{2}\right\rangle, \ldots,\left\langle e_{1}, \ldots, e_{n}\right\rangle\right)\right\} \\
& =\left\{\left(V_{1,0}, \ldots, V_{n, 0}\right)\right\} \\
& =\left\{V_{\bullet} \in Y_{n}: X V_{i} \subset V_{i} \text { and } V_{n}=V_{n, 0}\right\}=Y_{n, 0} .
\end{aligned}
$$

Thus we have proven $C_{\boldsymbol{a}, n, 0}$ is diffeomorphic to $Y_{n, 0}$ for all $n$. There is a unique noncrossing matching $\boldsymbol{a} \in B^{1,1}$ namely the one with arc (1,2). From [Russell and Tymoczko 2008, Lemma 5.3] $C_{\boldsymbol{a}, 1,1}$ is diffeomorphic to $Y_{1,1}$.

We proceed by induction assuming the statement is true for $(n-k-1, k-1)$ in order to prove it for $(n-k, k)$. Assume that the claim holds for $(n-k-1, k-1)$ 
where $k \geq 1$. Let $\boldsymbol{a} \in B^{n-k, k}$ be a matching of type $(n-k, k)$. Because $\boldsymbol{a}$ is noncrossing, it necessarily has an arc of the form $(i, i+1)$. Let $\boldsymbol{a}^{\prime} \in B^{n-k-1, k-1}$ be the matching of type $(n-k-1, k-1)$ obtained by erasing the arc $(i, i+1)$ in $\boldsymbol{a}$. Then we have the $\mathbb{P}^{1}$ bundle $C_{\boldsymbol{a}, n, k} \stackrel{q}{\rightarrow} C_{\boldsymbol{a}^{\prime}, n-k-1, k-1}$.

If $q\left(V_{\bullet}\right)=V_{\bullet}^{\prime}$ then for each $j$ there exists some $m$ with $V_{j} \subseteq X^{-1} V_{m}^{\prime}$. Since $V_{\bullet}^{\prime}=\left(V_{1}^{\prime}, \ldots, V_{n-2}^{\prime}\right)=\left(V_{1}, \ldots, V_{i-1}, X V_{i+2}, \ldots, X V_{n}\right)$ we may choose $m=j$ for $j \leq i, m=i$ for $i<j<i+3$, and $m=i-2$ for $j>i+2$.

Since $V_{\bullet}^{\prime} \in C_{\boldsymbol{a}^{\prime}, n-k-1, k-1}$ each $V_{m}^{\prime}$ is contained in $V_{n-k-1, k-1}$, so each $V_{j}$ is contained in $X^{-1} V_{n-k-1, k-1}=V_{n-k, k}$. Thus for all noncrossing matchings $\boldsymbol{a} \in$ $B^{n-k, k}$, each $C_{a, n-k, k}$ is contained in $Y_{n-k, k}$. Since $\widetilde{\Phi}^{-1} \circ \ell$ is a diffeomorphism, all the $C_{\boldsymbol{a}, n-k, k}$ are compact irreducible subvarieties of $Y_{n-k, k}$ with the same dimension as $X_{n-k, k}^{\prime}$. Thus each $C_{a, n-k, k}$ is an irreducible component of the Springer variety $Y_{n-k, k}$. Since $S_{\boldsymbol{a}, n-k, k}^{\prime} \neq S_{\boldsymbol{b}, n-k, k}^{\prime}$ for $\boldsymbol{a} \neq \boldsymbol{b}$ and $\widetilde{\Phi} \circ \boldsymbol{\ell}$ is a diffeomorphism, it follows that $C_{\boldsymbol{a}, n-k, k} \neq C_{\boldsymbol{b}, n-k, k}$ for $\boldsymbol{a} \neq \boldsymbol{b}$.

Recall that the irreducible components of $\mathscr{S}_{n-k, k}$ are in bijection with standard Young tableaux of shape $(n-k, k)$. [Stroppel and Webster 2010, Proposition 1.3] shows the set of noncrossing matchings of type $(n-k, k)$ are in bijection with standard Young tableaux of shape $(n-k, k)$. These results together show that the irreducible components of $\mathscr{S}_{n-k, k}$ are indexed by noncrossing matchings of type $(n-k, k)$. Thus $\bigcup_{\boldsymbol{a} \in B^{n-k, k}} C_{\boldsymbol{a}, n-k, k} \subseteq Y_{n-k, k}$ is isomorphic to the $(n, n-k)$ Springer variety, and we conclude $\bigcup_{\boldsymbol{a} \in B^{n-k, k}} C_{\boldsymbol{a}, n-k, k}=Y_{n-k, k}$.

Theorem 2.7. The $(n, n-k)$ Springer variety $\mathscr{S}_{n-k, k}$ is homeomorphic to $X_{n-k, k}$.

Proof. Lemmas 2.5 and 2.6 imply that $\mathscr{Y}_{n-k, k}$ is homeomorphic to $X_{n-k, k}^{\prime}$. Now we use an antipodal map to show that $X_{n-k, k}^{\prime}$ and $X_{n-k, k}$ are homeomorphic.

Define $\gamma:\left(S^{2}\right)^{n} \rightarrow\left(S^{2}\right)^{n}$ as $\gamma\left(\left(x_{1}, \ldots, x_{n}\right)\right)=\left(-x_{1}, x_{2}, \ldots,(-1)^{n} x_{n}\right)$. This map is its own inverse and thus is a diffeomorphism. Since $\boldsymbol{a} \in B^{n-k, k}$ is noncrossing each arc in $\boldsymbol{a}$ has some number of arcs and no rays between its endpoints. This means that there are an even number of vertices between the endpoints of each arc and every $\operatorname{arc}(i, j) \in \boldsymbol{a}$ has one even and one odd endpoint. Therefore $\gamma$ is the identity on exactly one of the coordinates $x_{i}, x_{j}$ and the antipodal map on the other. Furthermore given a ray $(i) \in \boldsymbol{a}$ the map $\gamma$ is the identity on $x_{i}$ if $i$ is even and the antipodal map if $i$ is odd. Hence $\gamma\left(X_{n-k, k}\right)=X_{n-k, k}^{\prime}$.

Again we alert the reader to the fact that this is a homeomorphism of topological spaces and not an isomorphism of algebraic varieties. One immediate consequence of our topological construction of the space $X_{n-k, k}$ is that given any two $\boldsymbol{a}, \boldsymbol{b} \in$ $B^{n-k, k}$ the spaces $S_{\boldsymbol{a}}$ and $S_{\boldsymbol{b}}$ are homeomorphic to the product $\left(S^{2}\right)^{k}$. Contrast this with [Stroppel and Webster 2010, Example 8] where the authors show that the two irreducible components of the $(2,2)$ Springer variety are both iterated $\mathbb{P}^{1}$-bundles but are not isomorphic as algebraic varieties. 
We finish this section by using our new construction to get a topological picture of the $(3,1)$ Springer variety. The topological model $X_{3,1}$ for the $(3,1)$ Springer variety is built from the homeomorphic images of three irreducible components indexed by the three elements of $B^{3,1}$ shown here:

$$
\boldsymbol{a}=\bigcap|| \boldsymbol{b}=|\bigcap| \boldsymbol{c}=|| \cap
$$

Each of these is homeomorphic to $S^{2}$ :

$$
\begin{aligned}
& S_{a}=\left\{(x, x,-p, p): x \in S^{2}\right\}, \\
& S_{b}=\left\{(-p, x, x, p): x \in S^{2}\right\}, \\
& S_{c}=\left\{(-p, p, x, x): x \in S^{2}\right\} .
\end{aligned}
$$

These spaces intersect in two different points:

$$
\begin{aligned}
& S_{a} \cap S_{b}=\left\{(-p,-p,-p, p) \in\left(S^{2}\right)^{4}\right\}, \\
& S_{b} \cap S_{c}=\left\{(-p, p, p, p) \in\left(S^{2}\right)^{4}\right\}, \\
& S_{\boldsymbol{a}} \cap S_{\boldsymbol{c}}=\varnothing .
\end{aligned}
$$

Putting this information together we see that $X_{3,1}$ is the wedge of three spheres as shown in Figure 2. Note that $H_{0}\left(X_{3,1}\right)=\mathbb{C}$ and that $H_{2}\left(X_{3,1}\right)=\mathbb{C}^{\left|B^{3,1}\right|}=\mathbb{C}^{3}$.

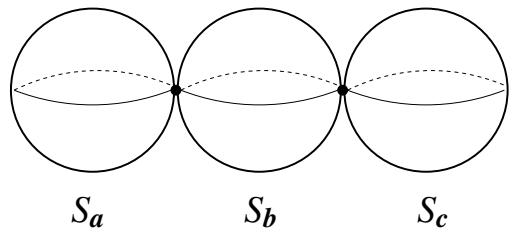

Figure 2. $X^{3,1}$ is a wedge of three copies of $S^{2}$.

\section{Intersections of components}

From now on we regard $X_{n-k, k}$ as the $(n-k, k)$ Springer variety. We reiterate that these two objects are the same in the topological sense and not the algebrogeometric sense. For fixed $n$ and $k$ we refer to $S_{\boldsymbol{a}, n-k, k} \operatorname{simply}$ as $S_{\boldsymbol{a}}$. We call these $S_{\boldsymbol{a}}$ which are the homeomorphic images of the irreducible components of the Springer variety the irreducible components (or components) of $X_{n-k, k}$.

This section follows [Khovanov 2004, Section 3] proving a sequence of lemmas about intersection between components and uses this information to set up a Mayer-Vietoris type exact sequence on homology. Proposition 3.9 and the $(m, m)$ case of Lemma 3.14 are stated without proof in that reference. Because we need these results for our arguments, we include proofs in the Appendix. Fung [2003] 
and Stroppel and Webster [2010] have studied intersections of the components of the Springer variety in the two-row case. Much of what we do in this section rediscovers their results via our topological model $X_{n-k, k}$.

\section{Matchings and their associated components.}

Definition 3.1. Given $\boldsymbol{a}, \boldsymbol{b} \in B^{n-k, k}$ let $\boldsymbol{a} w(\boldsymbol{b})$ be the result of reflecting $\boldsymbol{b}$ horizontally and gluing this reflection to $\boldsymbol{a}$, as in this example:

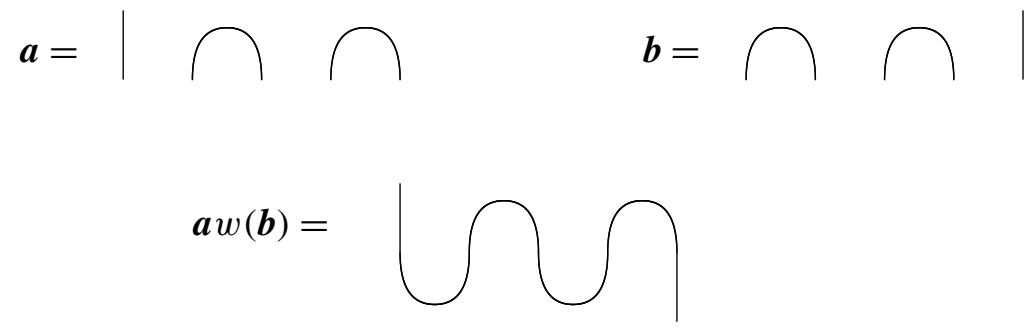

The 1-manifold $\boldsymbol{a} w(\boldsymbol{b})$ will consist of circles, lines with both endpoints pointing up, lines with both endpoints pointing down, and lines with one endpoint pointing in each direction. We define $|\boldsymbol{a} w(\boldsymbol{b})|$ to be the number of connected components in $\boldsymbol{a} w(\boldsymbol{b})$.

Definition 3.2 (order on noncrossing matchings). Given $\boldsymbol{a}, \boldsymbol{b} \in B^{n-k, k}$, write $\boldsymbol{a} \rightarrow \boldsymbol{b}$ if one of the following is true:

- There is a quadruple $i<j<k<l$ such that $(i, j),(k, l) \in \boldsymbol{a},(i, l),(j, k) \in \boldsymbol{b}$, and $\boldsymbol{a}$ and $\boldsymbol{b}$ are identical on all vertices not equal to $i, j, k, l$.

- There is a triple $i<j<k$ where $(i),(j, k) \in \boldsymbol{a},(i, j),(k) \in \boldsymbol{b}$, and $\boldsymbol{a}$ and $\boldsymbol{b}$ are identical on all vertices not equal to $i, j, k$.

Define a partial order $\boldsymbol{a} \prec \boldsymbol{b}$ if there exist a chain of arrows $\boldsymbol{a} \rightarrow \cdots \rightarrow \boldsymbol{b}$. Extend this to a total order $<$. Note that the extension to a total order is not unique. Figure 3 shows an example.

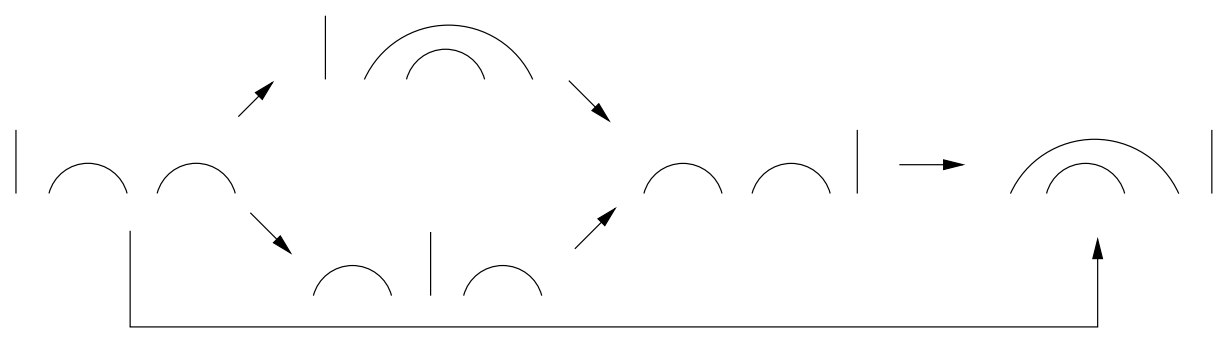

Figure 3. The $\rightarrow$ relation on elements of $B^{3,2}$. 
Definition 3.3. Given $\boldsymbol{a}, \boldsymbol{b} \in B^{n-k, k}$ let the distance from $\boldsymbol{a}$ to $\boldsymbol{b}$, denoted $d(\boldsymbol{a}, \boldsymbol{b})$, be the minimal length $m$ of a sequence $\left(\boldsymbol{a}=\boldsymbol{a}_{0}, \boldsymbol{a}_{1}, \ldots, \boldsymbol{a}_{m-1}, \boldsymbol{a}_{m}=\boldsymbol{b}\right)$ such that $\boldsymbol{a}_{i} \rightarrow \boldsymbol{a}_{i+1}$ or $\boldsymbol{a}_{i} \leftarrow \boldsymbol{a}_{i+1}$ for all $i$. We call a sequence that realizes the distance between $\boldsymbol{a}$ and $\boldsymbol{b}$ a minimal sequence.

The following observation of Fung shows that the line segments in the one manifold $\boldsymbol{a} w(\boldsymbol{b})$ tell us when two components have empty intersection. A refinement of this result given in [Stroppel and Webster 2010, Lemma 19] further characterizes the intersection of components by looking at orientations of the 1-manifold $\boldsymbol{a} w(\boldsymbol{b})$.

Proposition 3.4 [Fung 2003, Theorem 7.3]. Given $\boldsymbol{a}, \boldsymbol{b} \in B^{n-k, k}$ if $\boldsymbol{a} w(\boldsymbol{b})$ has a line with both endpoints pointing in the same direction then $S_{a} \cap S_{b}=\varnothing$.

We give a special name to the subset of $B^{n-k, k}$ consisting of matchings associated to components that have nonempty intersection with a specific component.

Definition 3.5. Given $\boldsymbol{a} \in B^{n-k, k}$ define the subset $B_{\boldsymbol{a}}^{n-k, k}$ of $B^{n-k, k}$ as

$$
B_{\boldsymbol{a}}^{n-k, k}=\left\{\begin{array}{r}
\boldsymbol{b} \in B^{n-k, k}: \text { each line in } \boldsymbol{a} w(\boldsymbol{b}) \text { has endpoints } \\
\text { pointing in opposite directions }
\end{array}\right\} .
$$

Applying Proposition 3.4 we see that given $\boldsymbol{a} \in B^{n-k, k}$

$$
\left(\bigcup_{\substack{\boldsymbol{b}<B^{n-k, k}}} S_{\boldsymbol{b}}\right) \cap S_{\boldsymbol{a}}=\left(\bigcup_{\substack{\boldsymbol{b}<\boldsymbol{a} \\ \boldsymbol{b} \in B_{\boldsymbol{a}}^{n-k, k}}} S_{\boldsymbol{b}}\right) \cap S_{\boldsymbol{a}} .
$$

For $\boldsymbol{b} \in B_{\boldsymbol{a}}^{n-k, k}$ the intersection $S_{\boldsymbol{a}} \cap S_{\boldsymbol{b}}$ is homeomorphic to $\left(S^{2}\right)^{\# \text { of circles in } \boldsymbol{a} w(\boldsymbol{b})}$.

Lemma 3.6. Let $\boldsymbol{a} \in B^{n-k, k}$ and $\boldsymbol{b} \in B_{\boldsymbol{a}}^{n-k, k}$. Then $\boldsymbol{a} w(\boldsymbol{b})$ is a union of circles and line segments. If we enumerate the rays in the matchings $\boldsymbol{a}$ and $\boldsymbol{b}$ from left to right as $i_{i}, \ldots, i_{n-2 k}$ and $j_{i}, \ldots, j_{2 n-k}$ respectively then in $\boldsymbol{a} w(\boldsymbol{b})$ each line segment will contain rays $i_{t}$ and $j_{t}$ for some $1 \leq t \leq n-2 k$.

Proof. Each line segment in $\boldsymbol{a} w(\boldsymbol{b})$ consists of two rays connected by some number of arcs. By assumption one ray is in $\boldsymbol{a}$ and one ray is in $\boldsymbol{b}$. Say that rays $i_{t_{1}}$ and $j_{t_{2}}$ are part of the same segment in $\boldsymbol{a} w(\boldsymbol{b})$. Then the rays $i_{i}, \ldots, i_{t_{1}-1}, j_{1}, \ldots, j_{t_{2}-1}$ lie on one side of this line segment. Since $\boldsymbol{a} w(\boldsymbol{b})$ is noncrossing these rays must be connected pairwise in a noncrossing manner. Since $\boldsymbol{b} \in B_{\boldsymbol{a}}^{n-k, k}$ each $i$-ray must be connected to a $j$-ray. Therefore $t_{1}=t_{2}$.

Lemma 3.7. Let $\boldsymbol{a} \in B^{n-k, k}$ and $\boldsymbol{b} \in B_{\boldsymbol{a}}^{n-k, k}$, and consider a sequence

$$
\left(\boldsymbol{a}=\boldsymbol{a}_{0}, \boldsymbol{a}_{1}, \ldots, \boldsymbol{a}_{m-1}, \boldsymbol{a}_{m}=\boldsymbol{b}\right) .
$$

If $\left|\boldsymbol{a}_{i} w(\boldsymbol{b})\right|=\left|\boldsymbol{a}_{i+1} w(\boldsymbol{b})\right|-1$ for all $i$, the sequence is minimal. 
Proof. For each $0 \leq i \leq m-1$ either $\boldsymbol{a}_{i} \rightarrow \boldsymbol{a}_{i+1}$ or $\boldsymbol{a}_{i} \leftarrow \boldsymbol{a}_{i+1}$. Thus either an arc and a ray are changing position, two unnested arcs are replaced by two nested arcs, or two nested arcs are replaced by unnested arcs. Consider the associated sequence

$$
\left(\boldsymbol{a} w(\boldsymbol{b})=\boldsymbol{a}_{0} w(\boldsymbol{b}), \boldsymbol{a}_{1} w(\boldsymbol{b}), \ldots, \boldsymbol{a}_{m-1} w(\boldsymbol{b}), \boldsymbol{a}_{m} w(\boldsymbol{b})=\boldsymbol{b} w(\boldsymbol{b})\right) .
$$

Analyzing each possibility, one can check that $\left|\boldsymbol{a}_{i} w(\boldsymbol{b})\right|=\left|\boldsymbol{a}_{i+1} w(\boldsymbol{b})\right| \pm 1$. Moreover, given $\boldsymbol{a}_{i} w(\boldsymbol{b})$ and $\boldsymbol{a}_{i+1} w(\boldsymbol{b})$, if the arc-ray or arc-arc pair that change from $\boldsymbol{a}_{i}$ to $\boldsymbol{a}_{i+1}$ are part of the same connected component in $\boldsymbol{a}_{i} w(\boldsymbol{b})$ then $\boldsymbol{a}_{i+1} w(\boldsymbol{b})$ will have one more component because we are splitting one component into two. On the other hand, if they are part of different connected components in $\boldsymbol{a}_{i} w(\boldsymbol{b})$ then $\boldsymbol{a}_{i+1} w(\boldsymbol{b})$ will have one less component because we are joining two components.

The one manifold $\boldsymbol{b} w(\boldsymbol{b})$ has the maximum number of components possible, so $|\boldsymbol{a} w(\boldsymbol{b})| \leq|\boldsymbol{b} w(\boldsymbol{b})|$. Since each arrow move changes the number of components by 1 , no sequence from $\boldsymbol{a}$ to $\boldsymbol{b}$ can be shorter than $|\boldsymbol{b} w(\boldsymbol{b})|-|\boldsymbol{a} w(\boldsymbol{b})|$. The sequence given in the statement of the lemma has $|\boldsymbol{a} w(\boldsymbol{b})|=|\boldsymbol{b} w(\boldsymbol{b})|-m$. Since no shorter sequence can exist the given sequence is minimal.

Given $\boldsymbol{a}, \boldsymbol{b} \in B^{n-k, k}$ label the $k$ connected components of $\boldsymbol{a} w(\boldsymbol{b})$ with $c_{1}, \ldots, c_{k}$. Associated to each $c_{i}$ let

$$
C_{i}=\left\{1 \leq j \leq n: c_{i} \text { passes through vertex } j \text { in } \boldsymbol{a} w(\boldsymbol{b})\right\} .
$$

Let the collection of all of these sets be denoted by $\mathscr{C}_{\boldsymbol{a}, \boldsymbol{b}}=\left\{C_{i}\right\}$. A useful way to understand the distance between matchings is to study the sequence of collections $\left(\mathscr{C}_{\boldsymbol{a}, \boldsymbol{b}}, \ldots, \mathscr{C}_{\boldsymbol{b}, \boldsymbol{b}}\right)$ associated to a sequence $\left(\boldsymbol{a}=\boldsymbol{a}_{0}, \boldsymbol{a}_{1}, \ldots \boldsymbol{a}_{m-1}, \boldsymbol{a}_{m}=\boldsymbol{b}\right)$. Restating Lemma 3.7 in the language of the sets $\mathscr{C}_{\boldsymbol{a}}, \boldsymbol{b}$ we have Lemma 3.8.

Lemma 3.8. Let $\boldsymbol{a} \in B^{n-k, k}$ and $\boldsymbol{b} \in B_{\boldsymbol{a}}^{n-k, k}$, and consider a sequence $(\boldsymbol{a}=$ $\left.\boldsymbol{a}_{0}, \ldots, \boldsymbol{a}_{m}=\boldsymbol{b}\right)$. If for each $0 \leq i \leq m-1$ the vertices of the arc-ray or arcarc pair in $\boldsymbol{a}_{i}$ being changed when transitioning from $a_{i}$ to $a_{i+1}$ are part of a single set in $\mathscr{C}_{\boldsymbol{a}_{i}, \boldsymbol{b}}$ then the sequence is minimal.

The following formula, which we prove in the Appendix, is given in [Khovanov 2004, page 9].

Proposition 3.9. For $n=2 m$ and $\boldsymbol{a}, \boldsymbol{b} \in B^{m, m}$ the distance $d(\boldsymbol{a}, \boldsymbol{b})$ is equal to $m-|\boldsymbol{a} w(\boldsymbol{b})|$.

We will extend this formula to the distance between matchings of any type. In order to prove this we appeal to the notion of completing matchings of type $(n-k, k)$ to matchings of type $(n-k, n-k)$. This idea is also used in [Khovanov 2004, Section 5.4]. Completions are a natural way to see the set $B^{n-k, k}$ sitting inside of $B^{n-k, n-k}$. 
Definition 3.10 (completion). Given $\boldsymbol{a} \in B^{n-k, k}$ enumerate the rays in $\boldsymbol{a}$ from left to right by $\left(i_{1}\right),\left(i_{2}\right), \ldots,\left(i_{n-2 k}\right)$. Define the function

$$
\varphi: B^{n-k, k} \rightarrow B^{n-k, n-k}
$$

where $\varphi(\boldsymbol{a})$ is the matching with $\operatorname{arcs}$

- $(i+n-2 k, j+n-2 k)$ if $(i, j)$ is an arc in $\boldsymbol{a}$,

- $\left(n-2 k-t+1, i_{t}+n-2 k\right)$ if $\left(i_{t}\right)$ is a ray in $\boldsymbol{a}$.

In other words, we complete each ray in $\boldsymbol{a}$ to an arc by adding additional vertices to the left of the matching and connecting unanchored endpoints of the rays to these vertices in the only way that keeps the matching noncrossing. We call $\varphi(\boldsymbol{a})$ the completion of $\boldsymbol{a}$. Figure 4 has an example.

Let $\widetilde{B}^{n-k, n-k}$ be the subset of $B^{n-k, n-k}$ consisting of matchings with no arcs of the form $(i, j)$ for $0 \leq i<j \leq n-2 k$. There is a natural inverse operation to completion on this subset of $B^{n-k, n-k}$.

Definition 3.11 (restriction). Consider $\boldsymbol{a} \in \widetilde{B}^{n-k, n-k}$ Define the function

$$
\psi: \widetilde{B}^{n-k, n-k} \rightarrow B^{n-k, k}
$$

where $\psi(\boldsymbol{a})$ is the matching with

- $\operatorname{arcs}(i-n+2 k, j-n+2 k)$ if $(i, j)$ is an $\operatorname{arc}$ in $\boldsymbol{a}$ with $n-2 k<i$,

- rays $(j-n+2 k)$ if $(i, j)$ is an arc in $\boldsymbol{a}$ with $1 \leq i \leq n-2 k$.

In other words, we remove the first $n-2 k$ vertices of $\boldsymbol{a}$ so that each arc with left endpoint incident on one of those vertices becomes a ray. We call $\psi(\boldsymbol{a})$ the restriction of $\boldsymbol{a}$. Figure 4 has an example.

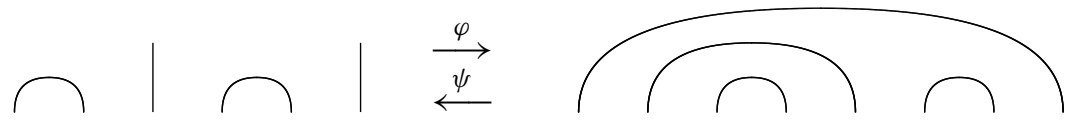

Figure 4. A matching $a \in B^{4,2}$ and its completion $\varphi(a) \in B^{4,4}$.

An immediate consequence of the definitions of restriction and completion is that $\varphi$ gives a one-to-one correspondence between the sets $B^{n-k, k}$ and $\widetilde{B}^{n-k, n-k}$. Note also that when $n=2 m$ and $k=m$ the sets $B^{n-k, k}, B^{n-k, n-k}$, and $\widetilde{B}^{n-k, n-k}$ are the same. Furthermore the maps $\varphi$ and $\psi$ are both the identity in this case.

Proposition 3.12. Given $\boldsymbol{a} \in B^{n-k, k}$ and $\boldsymbol{b} \in B_{\boldsymbol{a}}^{n-k, k}$, every minimal sequence $(\varphi(\boldsymbol{a}), \ldots, \varphi(\boldsymbol{b}))$ gives a minimal sequence $(\boldsymbol{a}, \ldots, \boldsymbol{b})$. 
Proof. Consider a minimal sequence $\left(\varphi(\boldsymbol{a})=\boldsymbol{x}_{0}, \boldsymbol{x}_{1}, \ldots, \boldsymbol{x}_{m-1}, \boldsymbol{x}_{m}=\varphi(\boldsymbol{b})\right)$. Since this sequence is minimal Lemma 3.8 guarantees that for all $0 \leq i<m$ the collection $\mathscr{C}_{\boldsymbol{x}_{i}, \varphi(\boldsymbol{b})}$ is a refinement of $\mathscr{C}_{\boldsymbol{x}_{i-1}, \varphi(\boldsymbol{b})}$ in the sense that every set in $\mathscr{C}_{\boldsymbol{x}_{i-1}, \varphi(\boldsymbol{b})}$ is a union of sets in $\mathscr{C}_{\boldsymbol{x}_{i}, \varphi(\boldsymbol{b})}$. In particular, $\mathscr{C}_{\boldsymbol{x}_{i}, \varphi(\boldsymbol{b})}$ contains exactly one more set than $\mathscr{C}_{\boldsymbol{x}_{i-1}, \varphi(\boldsymbol{b})}$.

By Lemma 3.6 we know that the $i$-th ray in $\boldsymbol{a}$ and the $i$-th ray in $\boldsymbol{b}$ are part of the same line segment in $\boldsymbol{a} w(\boldsymbol{b})$. Under $\varphi$ this line segment is completed to a circle passing through the newly added vertex $n-2 k-i+1$. Thus each set in $\mathscr{C}_{\varphi(\boldsymbol{a}), \varphi(\boldsymbol{b})}$ contains at most one of the vertices $1, \ldots, n-2 k$. Indeed the sets coming from circles in $\varphi(\boldsymbol{a}) w(\varphi(\boldsymbol{b}))$ that were line segments in $\boldsymbol{a} w(\boldsymbol{b})$ each contain one of these vertices.

Each set in $\mathscr{C}_{\boldsymbol{x}_{i}, \varphi(\boldsymbol{b})}$ is a refinement of the collection $\mathscr{C}_{\varphi(\boldsymbol{a}), \varphi(\boldsymbol{b})}$, so the sets in $\mathscr{C}_{\boldsymbol{x}_{i}, \varphi(\boldsymbol{b})}$ also each contain at most one of the vertices $1, \ldots, n-2 k$. This means that for all $0 \leq i \leq m$ the arcs in the matching $\boldsymbol{x}_{i}$ have at most one vertex incident on the vertices $1, \ldots, n-2 k$. Therefore $\boldsymbol{x}_{i} \in \widetilde{B}^{n-k, n-k}$. We can further conclude that whenever $\boldsymbol{x}_{i} \rightarrow \boldsymbol{x}_{i+1}$ then $\psi\left(\boldsymbol{x}_{i}\right) \rightarrow \psi\left(\boldsymbol{x}_{i+1}\right)$. Similarly whenever $\boldsymbol{x}_{i} \leftarrow \boldsymbol{x}_{i+1}$ then $\psi\left(\boldsymbol{x}_{i}\right) \leftarrow \psi\left(\boldsymbol{x}_{i+1}\right)$.

We conclude that the sequence $\left(\varphi(\boldsymbol{a})=\boldsymbol{x}_{0}, \ldots, \boldsymbol{x}_{m}=\varphi(\boldsymbol{b})\right)$ has a corresponding sequence $\left(\boldsymbol{a}, \psi\left(\boldsymbol{x}_{1}\right), \ldots, \psi\left(\boldsymbol{x}_{m-1}\right), \boldsymbol{b}\right)$. Finally, using the formula in Proposition 3.9 we see that

$$
d(\varphi(\boldsymbol{a}), \varphi(\boldsymbol{b}))=n-k-|\varphi(\boldsymbol{a}) w(\varphi(\boldsymbol{b}))|=n-k-|\boldsymbol{a} w(\boldsymbol{b})| .
$$

Thus we conclude that this sequence is minimal.

Corollary 3.13. Let $\boldsymbol{a} \in B^{n-k, k}$ and $\boldsymbol{b} \in B_{\boldsymbol{a}}^{n-k, k}$. Then $d(\boldsymbol{a}, \boldsymbol{b})=n-k-|\boldsymbol{a} w(\boldsymbol{b})|$.

Next we want to show that a very specific minimal sequence between $\boldsymbol{a}$ and $\boldsymbol{b}$ always exists.

Lemma 3.14. Given $\boldsymbol{a} \in B^{n-k, k}$ and $\boldsymbol{b} \in B_{\boldsymbol{a}}^{n-k, k}$ there exists $\boldsymbol{c} \in B_{\boldsymbol{a}}^{n-k, k}$ with $d(\boldsymbol{a}, \boldsymbol{b})=d(\boldsymbol{a}, \boldsymbol{c})+d(\boldsymbol{c}, \boldsymbol{b})$ and $\boldsymbol{a} \succ \boldsymbol{c} \prec \boldsymbol{b}$.

Proof. [Khovanov 2004, Lemma 1] asserts this statement for the $(m, m)$ case. This proof, which is fairly involved, is given in the Appendix.

Using this fact we can guarantee the existence of a minimal sequence

$$
\varphi(\boldsymbol{a}) \leftarrow \boldsymbol{a}_{1} \leftarrow \cdots \leftarrow \boldsymbol{c}^{\prime} \rightarrow \cdots \rightarrow \boldsymbol{a}_{m-1} \rightarrow \varphi(\boldsymbol{b}) .
$$

The proof of Proposition 3.12 implies that for all $1 \leq i \leq m$ we have $\psi\left(\boldsymbol{a}_{i}\right) \in B_{\boldsymbol{a}}^{n-k, k}$ and $\psi\left(\boldsymbol{a}_{i}\right) \in B_{\boldsymbol{b}}^{n-k, k}$. This gives us the minimal sequence

$$
\boldsymbol{a} \leftarrow \psi\left(\boldsymbol{a}_{1}\right) \leftarrow \cdots \leftarrow \psi\left(\boldsymbol{c}^{\prime}\right) \rightarrow \cdots \rightarrow \psi\left(\boldsymbol{a}_{m-1}\right) \rightarrow \boldsymbol{b} .
$$

Setting $\psi\left(\boldsymbol{c}^{\prime}\right)=\boldsymbol{c}$ the lemma is proven. 
Lemma 3.15. Let $\boldsymbol{a} \in B^{n-k, k}$ and $\boldsymbol{b} \in B_{\boldsymbol{a}}^{n-k, k}$. If $d(\boldsymbol{a}, \boldsymbol{c})=d(\boldsymbol{a}, \boldsymbol{b})+d(\boldsymbol{b}, \boldsymbol{c})$ then

$$
S_{a} \cap S_{c}=S_{a} \cap S_{b} \cap S_{c} .
$$

Proof. Clearly $S_{\boldsymbol{a}} \cap S_{\boldsymbol{b}} \cap S_{\boldsymbol{b}} \subseteq S_{\boldsymbol{a}} \cap S_{\boldsymbol{c}}$.

Since $d(\boldsymbol{a}, \boldsymbol{c})=d(\boldsymbol{a}, \boldsymbol{b})+d(\boldsymbol{b}, \boldsymbol{c})$, there is a minimal sequence $(\boldsymbol{a}, \ldots, \boldsymbol{b}, \ldots, \boldsymbol{c})$. Consider the collections $\mathscr{C}_{\boldsymbol{a}, \boldsymbol{c}}=\mathscr{C}_{\boldsymbol{c}, \boldsymbol{a}}=\left\{C_{1}, \ldots, C_{i_{\boldsymbol{a}, \boldsymbol{c}}}\right\}, \mathscr{C}_{\boldsymbol{a}, \boldsymbol{b}}=\left\{C_{1}^{\prime}, \ldots, C_{\boldsymbol{i}_{\boldsymbol{a}, \boldsymbol{b}}}^{\prime}\right\}$, and $\mathscr{C}_{\boldsymbol{b}, \boldsymbol{c}}=\left\{C_{1}^{\prime \prime}, \ldots, C_{\boldsymbol{i}_{\boldsymbol{b}, \boldsymbol{c}}}^{\prime \prime}\right\}$. The minimal sequence ensures that the collections $\mathscr{C}_{\boldsymbol{a}, \boldsymbol{b}}$ and $\mathscr{C}_{\boldsymbol{b}, \boldsymbol{c}}$ are each refinements of the collection $\mathscr{C}_{\boldsymbol{a}, \boldsymbol{c}}$.

In terms of sets in $\mathscr{C}_{\boldsymbol{a}, \boldsymbol{c}}$ we have

$$
S_{\boldsymbol{a}} \cap S_{\boldsymbol{c}}=\left\{x=\left(x_{1}, \ldots, x_{n}\right): x_{i}=x_{j} \text { if there is } C_{k} \in \mathscr{C}_{\boldsymbol{a}, \boldsymbol{c}} \text { with } i, j \in C_{k}\right\} \subset S_{\boldsymbol{a}} .
$$

Whenever $i, j \in C_{k}$ there are some $C_{k_{1}}^{\prime} \in \mathscr{C}_{\boldsymbol{a}, \boldsymbol{b}}$ and $C_{k_{2}} \in \mathscr{C}_{\boldsymbol{b}, \boldsymbol{c}}$ with $C_{k} \subset C_{k_{1}}^{\prime}$ amd $C_{k} \subset C_{k_{2}}^{\prime \prime}$. Then $x \in S_{\boldsymbol{a}} \cap S_{\boldsymbol{b}}$ and $x \in S_{\boldsymbol{b}} \cap S_{\boldsymbol{c}}$. Finally we conclude that $S_{\boldsymbol{a}} \cap S_{\boldsymbol{c}} \subseteq S_{\boldsymbol{a}} \cap S_{\boldsymbol{b}} \cap S_{\boldsymbol{c}}$

Lemma 3.16. Let $S_{<\boldsymbol{a}} \cap S_{\boldsymbol{a}}=\left(\cup_{\boldsymbol{b}<\boldsymbol{a}} S_{\boldsymbol{b}}\right) \cap S_{\boldsymbol{a}}$. Then $S_{<\boldsymbol{a}} \cap S_{\boldsymbol{a}}=\cup_{\boldsymbol{b} \rightarrow \boldsymbol{a}}\left(S_{\boldsymbol{b}} \cap S_{\boldsymbol{a}}\right)$.

Proof. By basic set theory we have $S_{<\boldsymbol{a}} \cap S_{\boldsymbol{a}}=\cup_{\boldsymbol{b}<\boldsymbol{a}}\left(S_{\boldsymbol{a}} \cap S_{\boldsymbol{b}}\right)$. Since $\boldsymbol{b}<\boldsymbol{a}$ whenever $\boldsymbol{b} \rightarrow \boldsymbol{a}$ we conclude that $\cup_{\boldsymbol{b} \rightarrow \boldsymbol{a}}\left(S_{\boldsymbol{b}} \cap S_{\boldsymbol{a}}\right) \subseteq S_{<\boldsymbol{a}} \cap S_{\boldsymbol{a}}$.

Take $x \in S_{<\boldsymbol{a}} \cap S_{\boldsymbol{a}}$. Then there exists $\boldsymbol{b}<\boldsymbol{a}$ such that $x \in S_{\boldsymbol{a}} \cap S_{\boldsymbol{b}}$. By Lemma 3.14 we have a minimal sequence $\left(\boldsymbol{a} \leftarrow \boldsymbol{a}_{1} \cdots \leftarrow \boldsymbol{c} \rightarrow \cdots \boldsymbol{a}_{m-1} \rightarrow \boldsymbol{b}\right)$. This sequence must begin with $\leftarrow$ since otherwise we would have a minimal sequence consisting entirely of $\rightarrow$ moves. The existence of such a sequence would mean that $\boldsymbol{a}<\boldsymbol{b}$, but we have assumed $\boldsymbol{b}<\boldsymbol{a}$.

From the sequence we have $d(\boldsymbol{a}, \boldsymbol{b})=d\left(\boldsymbol{a}, \boldsymbol{a}_{1}\right)+d\left(\boldsymbol{a}_{1}, \boldsymbol{b}\right)$. From Lemma 3.15 we know

$$
S_{\boldsymbol{a}} \cap S_{\boldsymbol{b}}=S_{\boldsymbol{a}} \cap S_{\boldsymbol{a}_{1}} \cap S_{\boldsymbol{b}} \subseteq S_{\boldsymbol{a}} \cap S_{\boldsymbol{a}_{1}} .
$$

We have shown that whenever $\boldsymbol{b}<\boldsymbol{a}$ there exists $\boldsymbol{a}_{1} \rightarrow \boldsymbol{a}$ with $S_{\boldsymbol{a}} \cap S_{\boldsymbol{b}} \subseteq S_{\boldsymbol{a}} \cap S_{\boldsymbol{a}_{1}}$.

A useful cell decomposition. We recall the definition of a CW-decomposition, which we will also sometimes call a cell decomposition or simply a decomposition. Then we introduce a CW-decomposition of $X_{n-k, k}$ that generalizes the one found in [Khovanov 2004, Lemma 4].

Definition 3.17. A CW-decomposition of a space $X$ is a partition of $X$ into open cells (possibly of varying dimension) each of which is either homeomorphic to an open ball (when the cell has dimension larger than zero) or a single point (when the cell has dimension zero).

Additionally it is required that for each $n$ dimensional open cell $C$ there is a homeomorphism $f$ from the closed ball of dimension $n$ to $X$ such that

- $f$ restricted to the interior of the closed ball is a homeomorphism onto $C$, and 
- the image under $f$ of the boundary of the closed ball is a finite union of cells of lower dimension.

As an example we can choose any point in the two-sphere $p \in S^{2}$. Then $p$ and $S^{2}-p$ constitute a CW-decomposition of $S^{2}$ into one two-dimensional cell and one zero-dimensional cell. Since each component $S_{\boldsymbol{a}} \in X_{n-k, k}$ is homeomorphic to a product of spheres, the components $S_{a}$ have an obvious CW-decomposition into even dimensional cells coming from their cartesian product structure. In Section 4 we will explore the combinatorics of this decomposition in depth.

This section gives another CW-decomposition for the components of $X_{n-k, k}$. This decomposition makes use of the order on matchings given in the previous section and restricts to a $\mathrm{CW}$-decomposition of intersections of certain components (see Lemma 3.20), making it easier to construct an iterated Mayer-Vietoris sequence on homology.

It is an interesting question what the relationship might be between the aforementioned CW-decompositions for components of $X_{n-k, k}$ and the Schubert cell decomposition of Grassmannians. A related issue is what connection the order defined in this paper on components has to do with the Bruhat order on Schubert cells. We are also interested in exploring the relationship between our cells and the attracting cells found in the paper [Stroppel and Webster 2010] on two-row Springer fibers. We hope to explore these connections in upcoming work.

Definition 3.18 (decomposition of $S_{a}$ ). Given a matching $\boldsymbol{a} \in B^{n-k, k}$ we construct a graph $\Gamma=(V, E)$ with a vertex $v \in V$ for each arc in $\boldsymbol{a}$. Given $v \in V$ let $\left(v_{1}, v_{2}\right)$ with $v_{1}<v_{2}$ be the arc in $a$ corresponding to $v$. There is an edge between $v, w \in V$ if and only if there is some $\boldsymbol{b} \in B^{n-k, k}$ with $\boldsymbol{b} \rightarrow \boldsymbol{a}$ where $\boldsymbol{b}$ is identical to $\boldsymbol{a}$ off of vertices $v_{1}, v_{2}, w_{1}, w_{2}$. In $\boldsymbol{b}$ these vertices are connected in the only other possible noncrossing manner.

Let $M$ be the set of all root vertices. Figure 5 has an example. Let $p=(0,0,1) \in$ $S^{2}$ once again be the north pole of the standard embedded unit sphere. We construct a set $c(J) \subset S_{a} \subset\left(S^{2}\right)^{n}$ where $J \subseteq(E \cup M)$.

Let $c(J) \subset\left\{x=\left(x_{1}, \ldots, x_{n}\right) \in S_{a}\right\}$ such that

- $x_{v_{1}}=x_{w_{1}}$ if edge $(v, w) \in J$,

- $x_{v_{1}} \neq x_{w_{1}}$ if edge $(v, w) \in E-J$,

- $x_{v_{1}}=(-1)^{v_{1}} p$ if vertex $v \in J$,

- $x_{v_{1}} \neq(-1)^{v_{1}} p$ if vertex $v \in M-J$.
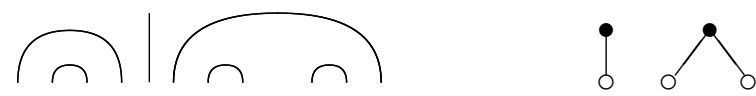

Figure 5. A matching and its graph $\Gamma$. 
Note that we only specify values for $x_{v_{1}}$ and $x_{w_{1}}$ because $x_{v_{1}}=x_{v_{2}}$ and $x_{w_{1}}=x_{w_{2}}$.

Lemma 3.19. The construction above is indeed a $C W$-decomposition of $S_{a}$.

Proof. These cells are products of the cells found in [Khovanov 2004] with some points in fixed positions. The CW-decomposition structure is thus inherited from the CW-decomposition structure on the cells in that reference.

One can also see this structure directly. Using induction on the number of arcs it is straightforward to prove that cells $c(J)$ are trivial $\mathbb{R}^{2}$ bundles over $\mathbb{R}^{2 k-|J|-2}$ and are thus homeomorphic to $\mathbb{R}^{2 k-|J|}$. The cells are disjoint by construction. Also by construction, the boundary of a cell is the union of smaller cells. More precisely, if we let $\mathscr{F}=\left\{J^{\prime} \subset(E \sqcup M): J \subsetneq J^{\prime}\right\}$ then $\partial(c(J))=\bigsqcup_{J^{\prime} \in \mathscr{\Phi}} c\left(J^{\prime}\right)$.

Lemma 3.20. Given $\boldsymbol{b} \rightarrow \boldsymbol{a}$ the cell decomposition for $S_{\boldsymbol{a}}$ restricts to a cell decomposition for $S_{\boldsymbol{a}} \cap S_{\boldsymbol{b}}$. Therefore the decomposition for $S_{\boldsymbol{a}}$ also restricts to a decomposition for $S_{<\boldsymbol{a}} \cap S_{\boldsymbol{a}}$.

Proof. Consider $\boldsymbol{b} \rightarrow \boldsymbol{a}$. Then either

- there exist $i<j<k<l$ with $(i, j),(k, l) \in \boldsymbol{b}$ and $(i, l),(j, k) \in \boldsymbol{a}$, or

- there exist $i<j<k$ with $(i),(j, k) \in \boldsymbol{b}$ and $(i, j),(k) \in \boldsymbol{a}$.

The matchings $\boldsymbol{a}$ and $\boldsymbol{b}$ are identical otherwise.

In the first case, no rays change position as we move from $\boldsymbol{b}$ to $\boldsymbol{a}$. Thus we get that $S_{\boldsymbol{a}} \cap S_{\boldsymbol{b}}$ is a subcomplex of $S_{\boldsymbol{a}}$ by [Khovanov 2004, Lemma 4].

Consider the second case. In order for both $\boldsymbol{a}$ and $\boldsymbol{b}$ to be noncrossing, there can be no $\operatorname{arcs}\left(i^{\prime}, k^{\prime}\right)$ or rays $\left(i^{\prime}\right)$ where $i<i^{\prime}<j<k<k^{\prime}$. Every arc with left endpoint $i^{\prime}$ with $i<i^{\prime}<j$ must have right endpoint $k^{\prime}$ with $k^{\prime}<j$, so all arcs incident on vertices between $i$ and $j$ are completely contained between $i$ and $j$. Therefore there is an even number of vertices between $i$ and $j$, and so $i$ and $j$ have opposite parity. Since there is an even number of vertices between $j$ and $k$ the vertices $i$ and $k$ have the same parity.

Let $\Gamma_{\boldsymbol{a}}=\left(V_{\boldsymbol{a}}, E_{\boldsymbol{a}}\right)$ with distinguished vertex set $M_{\boldsymbol{a}}$ be the graph for $\boldsymbol{a}$. The vertex $v \in V_{\boldsymbol{a}}$ corresponding to the $\operatorname{arc}(i, j) \in \boldsymbol{a}$ is a root, so $v \in M_{\boldsymbol{a}}$. Given $J \subset\left(V_{\boldsymbol{a}} \cup M_{\boldsymbol{a}}\right)$ whenever $v \in J$ all points $x=\left(x_{1}, \ldots, x_{n}\right) \in c(J)$ have $x_{i}=x_{j}=$ $(-1)^{i} p$. For all $J$ and for all $x \in c(J)$ we also have $x_{k}=(-1)^{k} p=(-1)^{i} p$ since $(k)$ is a ray in $\boldsymbol{a}$. Since $S_{\boldsymbol{a}} \cap S_{\boldsymbol{b}}=\left\{x \in S_{\boldsymbol{a}}: x_{i}=x_{j}=x_{k}=(-1)^{i} p\right\}$ the cells $c(J)$ for which $v \in J$ form a cell complex for $S_{\boldsymbol{a}} \cap S_{\boldsymbol{b}}$.

Appealing to Lemma 3.16 we see that $S_{<a} \cap S_{a}$ is a subcomplex of the complex for $S_{a}$.

Building an exact sequence. The following two statements are consequences of Lemma 3.16. 
Lemma 3.21. The inclusion $\left(S_{<\boldsymbol{a}} \cap S_{\boldsymbol{a}}\right) \hookrightarrow S_{\boldsymbol{a}}$ induces an injective homomorphism on homology $H_{*}\left(S_{<\boldsymbol{a}} \cap S_{\boldsymbol{a}}\right) \hookrightarrow H_{*}\left(S_{\boldsymbol{a}}\right)$.

Lemma 3.22. The homomorphism $\bigoplus H_{*}\left(S_{\boldsymbol{b}} \cap S_{\boldsymbol{a}}\right) \stackrel{f^{\prime}}{\longrightarrow} H_{*}\left(S_{<\boldsymbol{a}} \cap S_{\boldsymbol{a}}\right)$ induced by inclusion is surjective. $\boldsymbol{b} \rightarrow \boldsymbol{a}$

Now consider the Mayer-Vietoris sequence:

$$
\cdots \rightarrow H_{m}\left(S_{<\boldsymbol{a}} \cap S_{\boldsymbol{a}}\right) \stackrel{f}{\longrightarrow} H_{m}\left(S_{<\boldsymbol{a}}\right) \oplus H_{m}\left(S_{\boldsymbol{a}}\right) \stackrel{g}{\longrightarrow} H_{m}\left(S_{\leq \boldsymbol{a}}\right) \rightarrow \cdots
$$

Lemma 3.23. The space $S_{\leq a}$ has nonzero homology only in even degrees. The Mayer-Vietoris sequence above reduces to short exact sequences of the form

$$
0 \rightarrow H_{2 m}\left(S_{<\boldsymbol{a}} \cap S_{\boldsymbol{a}}\right) \stackrel{f}{\longrightarrow} H_{2 m}\left(S_{<\boldsymbol{a}}\right) \oplus H_{2 m}\left(S_{\boldsymbol{a}}\right) \stackrel{g}{\longrightarrow} H_{2 m}\left(S_{\leq \boldsymbol{a}}\right) \rightarrow 0 .
$$

Proof. We prove this inductively with respect to the order $<$. The base case is obvious since for the first $\boldsymbol{a}$ in the order $S_{\leq \boldsymbol{a}}=S_{\boldsymbol{a}}$. Assume the lemma is true for all matchings up to and including some matching $\boldsymbol{e}$, and let $\boldsymbol{a}$ be the next matching in the order. Then $S_{<\boldsymbol{a}}=S_{\leq \boldsymbol{e}}, S_{\boldsymbol{a}}$, and $S_{<\boldsymbol{a}} \cap S_{\boldsymbol{a}}$ all have homology in even degrees only. Therefore the Mayer-Vietoris sequence decomposes into exact sequences $0 \rightarrow H_{2 m+1}\left(S_{\leq \boldsymbol{a}}\right) \stackrel{\partial}{\longrightarrow} H_{2 m}\left(S_{<\boldsymbol{a}} \cap S_{\boldsymbol{a}}\right) \stackrel{f}{\longrightarrow} H_{2 m}\left(S_{<\boldsymbol{a}}\right) \oplus H_{2 m}\left(S_{\boldsymbol{a}}\right) \stackrel{g}{\longrightarrow} H_{2 m}\left(S_{\leq \boldsymbol{a}}\right) \rightarrow 0$.

By Lemma 3.21 the map $H_{2 m}\left(S_{<\boldsymbol{a}} \cap S_{\boldsymbol{a}}\right) \rightarrow H_{2 m}\left(S_{\boldsymbol{a}}\right)$ is injective so $H_{2 m+1}\left(S_{\leq \boldsymbol{a}}\right)=$ 0 as desired.

Theorem 3.24. The sequence

$$
\bigoplus_{\boldsymbol{b} \rightarrow \boldsymbol{c} \leq \boldsymbol{a}} H_{*}\left(S_{\boldsymbol{b}} \cap S_{\boldsymbol{c}}\right) \stackrel{\psi^{-}}{\longrightarrow} \bigoplus_{\boldsymbol{b} \leq \boldsymbol{a}} H_{*}\left(S_{\boldsymbol{b}}\right) \stackrel{\phi}{\longrightarrow} H_{*}\left(S_{\leq \boldsymbol{a}}\right) \rightarrow 0
$$

is exact. Here $\psi^{-}$is defined to be $\Sigma_{\boldsymbol{b} \rightarrow \boldsymbol{c} \leq \boldsymbol{a}}\left(\psi_{\boldsymbol{b}, \boldsymbol{c}}-\psi_{\boldsymbol{c}, \boldsymbol{b}}\right)$ where $\psi_{\boldsymbol{b}, \boldsymbol{c}}: H_{*}\left(S_{\boldsymbol{b}} \cap S_{\boldsymbol{c}}\right) \rightarrow$ $H_{*}\left(S_{\boldsymbol{b}}\right)$ is induced by the inclusion $S_{\boldsymbol{b}} \cap S_{\boldsymbol{c}} \subset S_{\boldsymbol{b}}$ and $\psi_{\boldsymbol{c}, \boldsymbol{b}}$ is similarly defined. The map $\phi$ is induced by the inclusions $S_{\boldsymbol{b}} \subset S_{\leq a}$.

Proof. This proof follows the structure of the proof of [Khovanov 2004, Proposition 4]. We prove this result by induction with respect to the total order on the set $B^{n-k, k}$. Let $\boldsymbol{a}_{0}$ be the first element with respect to this order. Then there do not exist $\boldsymbol{b}, \boldsymbol{c} \leq \boldsymbol{a}_{0}$ and $S_{\leq \boldsymbol{a}_{0}}=S_{\boldsymbol{a}_{0}}$. The sequence

$$
0 \rightarrow H_{*}\left(S_{\boldsymbol{a}_{0}}\right) \stackrel{\phi}{\longrightarrow} H_{*}\left(S_{\boldsymbol{a}_{0}}\right) \rightarrow 0
$$

is exact since $\phi$ is an isomorphism, and the base case is proven.

Assume for some element $\boldsymbol{a}_{i-1} \in B^{n-k, k}$ that the sequence

$$
\bigoplus_{\boldsymbol{b} \rightarrow \boldsymbol{c} \leq \boldsymbol{a}_{i-1}} H_{*}\left(S_{\boldsymbol{b}} \cap S_{\boldsymbol{c}}\right) \stackrel{\psi^{-}}{\longrightarrow} \bigoplus_{\boldsymbol{b} \leq \boldsymbol{a}_{i-1}} H_{*}\left(S_{\boldsymbol{b}}\right) \stackrel{\phi}{\longrightarrow} H_{*}\left(S_{\leq \boldsymbol{a}_{i-1}}\right) \rightarrow 0
$$


is exact. Consider the next element $\boldsymbol{a}_{i}$. By Lemma 3.23 we know that

$$
0 \rightarrow H_{*}\left(S_{<\boldsymbol{a}_{i}} \cap S_{\boldsymbol{a}_{i}}\right) \stackrel{f}{\longrightarrow} H_{*}\left(S_{<\boldsymbol{a}_{i}}\right) \oplus H_{*}\left(S_{\boldsymbol{a}_{i}}\right) \stackrel{g}{\longrightarrow} H_{*}\left(S_{\leq \boldsymbol{a}_{i}}\right) \rightarrow 0
$$

is an exact sequence. By Lemma 3.22 the map $f^{\prime}: \bigoplus_{\boldsymbol{b} \rightarrow \boldsymbol{c} \leq \boldsymbol{a}_{i}} H_{*}\left(S_{\boldsymbol{b}} \cap S_{\boldsymbol{c}}\right) \rightarrow$ $H_{*}\left(S_{<\boldsymbol{a}_{i}} \cap S_{\boldsymbol{a}_{i}}\right)$ is surjective so $\operatorname{Im}(f)=\operatorname{Im}\left(f \circ f^{\prime}\right)$. Thus we get the exact sequence

$$
\bigoplus_{\boldsymbol{b} \rightarrow \boldsymbol{c} \leq \boldsymbol{a}_{i}} H_{*}\left(S_{\boldsymbol{b}} \cap S_{\boldsymbol{c}}\right) \stackrel{f^{\prime} \circ f}{\longrightarrow} H_{*}\left(S_{<\boldsymbol{a}_{i}}\right) \oplus H_{*}\left(S_{\boldsymbol{a}_{i}}\right) \stackrel{g}{\longrightarrow} H_{*}\left(S_{\leq \boldsymbol{a}_{i}}\right) \rightarrow 0 .
$$

Now consider the sequence

$$
\bigoplus_{\boldsymbol{b} \rightarrow \boldsymbol{c} \leq \boldsymbol{a}_{i}} H_{*}\left(S_{\boldsymbol{b}} \cap S_{\boldsymbol{c}}\right) \stackrel{\psi^{-}}{\longrightarrow} \bigoplus_{\boldsymbol{b} \leq \boldsymbol{a}_{i}} H_{*}\left(S_{\boldsymbol{b}}\right) \stackrel{\phi}{\longrightarrow} H_{*}\left(S_{\leq \boldsymbol{a}_{i}}\right) \rightarrow 0 .
$$

Since the inclusions $S_{\boldsymbol{b}} \cap S_{\boldsymbol{c}} \subset S_{<\boldsymbol{a}_{i}} \subset S_{\leq \boldsymbol{a}_{i}}$ and $S_{\boldsymbol{b}} \cap S_{\boldsymbol{c}} \subset S_{\boldsymbol{b}} \subset S_{\leq \boldsymbol{a}_{i}}$ and $S_{\boldsymbol{b}} \cap S_{\boldsymbol{c}} \subset$ $S_{c} \subset S_{\leq a_{i}}$ all induce the same maps on homology we see that $\operatorname{Im}\left(\psi^{-}\right)=\operatorname{Ker}(\phi)$. Take some $y \in H_{*}\left(S_{\leq \boldsymbol{a}_{i}}\right)$. By exactness there exists $\left(x, x^{\prime}\right) \in H_{*}\left(S_{<\boldsymbol{a}_{i}}\right) \oplus H_{*}\left(S_{\boldsymbol{a}_{i}}\right)$ with $g\left(x, x^{\prime}\right)=y$. Again by exactness, there exists some $x^{\prime \prime} \in \bigoplus_{\boldsymbol{b} \leq \boldsymbol{a}_{i-1}} H_{*}\left(S_{\boldsymbol{b}}\right)$ with $\phi\left(x^{\prime \prime}\right)=x$. Then we have $\phi\left(x^{\prime \prime}, x^{\prime}\right)=g\left(x, x^{\prime}\right)=y$, and the desired sequence is exact.

\section{Diagrammatic homology generators}

In the last section we proved an exact sequence on the collection of complex vector spaces $H_{*}\left(X_{n-k, k}\right)$. In this section we will ultimately use this sequence to give diagrammatic bases for these vector spaces. We begin by giving a diagrammatic collection of generators and relations for $H_{*}\left(X_{n-k, k}\right)$.

Let $\boldsymbol{a}, \boldsymbol{b} \in B^{n-k, k}$ with $\boldsymbol{a} \rightarrow \boldsymbol{b}$. Recall that $S_{\boldsymbol{a}}$ is homeomorphic to $\left(S^{2}\right)^{k}$ and $S_{\boldsymbol{a}} \cap S_{\boldsymbol{b}}$ is homeomorphic to $\left(S^{2}\right)^{k-1}$. We will use the cartesian product CWdecomposition for $S_{\boldsymbol{a}}$ to give a convenient diagrammatic way to represent cells. This is a different decomposition than the one found in Section 3. While that decomposition was useful for constructing an exact sequence on homology, the cartesian product decomposition lends itself nicely to the diagrammatic calculations we would like to do in the remainder of this paper.

Let $E$ be the set of arcs in $a$, and for each $e \in E$ let $e_{\ell}$ be the left endpoint of the arc $e$ and $e_{r}$ be the right endpoint. Note that for each $x=\left(x_{1}, \ldots, x_{n}\right) \in S_{\boldsymbol{a}}$ we have $x_{e_{\ell}}=x_{e_{r}}$. Therefore to describe a subset of $S_{a}$ we only need to specify the value of $x_{e_{\ell}}$. Consider the cell decomposition of the two-sphere given by the point $p=(0,0,1) \in S^{2}$ and the two-cell $c=S^{2}-p$.

Definition 4.1 (diagrammatic homology basis for $S_{a}$ ). Given $I \subset E$ we define the following cell $c(I)$ which is homeomorphic to $\mathbb{R}^{2|I|}$.

$$
c(I)=\left\{x=\left(x_{1}, \ldots, x_{n}\right) \in S_{a}: x_{e_{\ell}}=p \text { if } e \notin I \text { and } x_{e_{\ell}} \neq p \text { if } e \in I\right\} .
$$


Varying over all possible subsets of $E$ we get the cartesian product cell decomposition for $S_{\boldsymbol{a}}$. Note that this decomposition is in general not the same as the one described in Definition 3.18. We can diagrammatically represent the cartesian product homology generators by considering certain markings of the matching $\boldsymbol{a}$.

Given $I \subset E$ let $M$ be the matching $\boldsymbol{a}$ decorated as follows:

- Mark each ray with a dot.

- For each $e \in I$ mark the corresponding arc in $\boldsymbol{a}$ with a dot.

- Leave all other arcs unmarked.

We call such a diagram a dotted noncrossing matching for $\boldsymbol{a}$. These will be denoted throughout by capital letters. Figure 6 has an example.

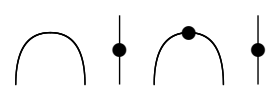

Figure 6. A dotted noncrossing matching $M$ for $\boldsymbol{a}$.

We use a similar diagrammatic convention to represent cartesian product cells for $\left(S^{2}\right)^{n}$ in [Russell and Tymoczko 2008, Definition 3.1]. These diagrams, which we call line diagrams, are collections of $n$ dotted and undotted vertical lines. The common theme of dotted noncrossing matchings and line diagrams is that unmarked lines and arcs correspond to two-cells while dotted lines and arcs correspond to points. We want to consider the behavior of these generators under maps induced by inclusion.

Definition 4.2. Consider $\boldsymbol{a} \rightarrow \boldsymbol{b} \in B^{n-k, k}$. Then either $\boldsymbol{a}$ and $\boldsymbol{b}$ agree off of two arcs or an arc and a ray. The first two relations deal with the case when $\boldsymbol{a}$ and $\boldsymbol{b}$ differ on two arcs. The third relation deals with the case when they differ on an arc and a ray.

Type I relations: Let $M_{1}$ be the dotted noncrossing matching with dotted $\operatorname{arc}(i, j)$ and undotted $\operatorname{arc}(k, l)$. Let $M_{2}$ be the dotted noncrossing matching with dotted arc $(k, l)$ and undotted arc $(i, j)$. Define $M_{1}^{\prime}$ and $M_{2}^{\prime}$ analogously for $\boldsymbol{b}$, so that they agree with the $M_{i}$ off of $i, j, k, l$. Type I relations have the form

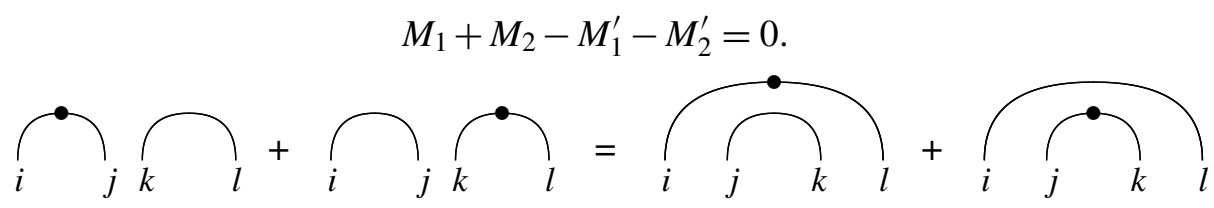

Figure 7. Type I relation. 
Type II relations: Let $M_{3}$ be the dotted noncrossing matching with dotted arcs $(i, j)$ and $(k, l)$. Define $M_{3}^{\prime}$ analogously for $\boldsymbol{b}$. Type II relations have the form

$$
M_{3}-M_{3}^{\prime}=0 .
$$

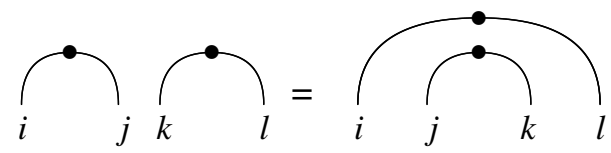

Figure 8. Type II relation.

Type III relations: Let $M_{4}$ be the dotted noncrossing matching with dotted ray $(i)$ and dotted arc $(j, k)$. Let $M_{4}^{\prime}$ be the dotted noncrossing matching identical to $M_{4}$ except with dotted arc $(i, j)$ and dotted ray $(k)$. Type III relations have the form

$$
M_{4}-M_{4}^{\prime}=0
$$

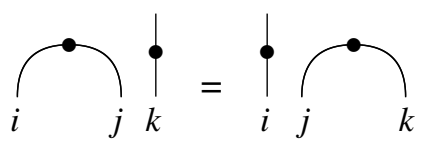

Figure 9. Type III relation.

Theorem 4.3. Let $\mathcal{T}$ be the subpace of $\bigoplus_{\boldsymbol{b}} H_{*}\left(S_{\boldsymbol{b}}\right)$ generated by all complex linear combinations of Type I, Type II, and Type III relations. Then there is the following isomorphism of complex vector spaces.

$$
H_{*}\left(X_{n-k, k}\right) \cong\left(\bigoplus_{\boldsymbol{b}} H_{*}\left(S_{\boldsymbol{b}}\right)\right) / \mathscr{T}
$$

Proof. From Theorem 3.24 we see that $\bigoplus_{\boldsymbol{b}} H_{*}\left(S_{\boldsymbol{b}}\right)$ generates $H_{*}\left(X_{n-k, k}\right)$ with relations coming from $\psi^{-}\left(H_{*}\left(S_{\boldsymbol{a}} \cap S_{\boldsymbol{b}}\right)\right)$ where $\boldsymbol{a} \rightarrow \boldsymbol{b}$. The following calculations show that these relations always have the form of Type I, II, or III relations. We use the notation for cartesian product cells found in Definition 4.1.

Consider $\boldsymbol{a}, \boldsymbol{b} \in B^{2,2}$ where $\boldsymbol{a}$ has $\operatorname{arcs}(1,2),(3,4)$ and $\boldsymbol{b}$ has $\operatorname{arcs}(1,4),(2,3)$. Then $\boldsymbol{a} w(\boldsymbol{b})$ consists of one circle, and $S_{\boldsymbol{a}} \cap S_{\boldsymbol{b}}$ is homeomorphic to $S^{2}$. Decompose $S_{a} \cap S_{b}$ into

$c_{1}=\left\{(p, p, p, p) \in S_{\boldsymbol{a}} \cap S_{\boldsymbol{b}}\right\} \cong \mathbb{R}^{0} \quad$ and $\quad c_{2}=\left\{(x, x, x, x) \in S_{\boldsymbol{a}} \cap S_{\boldsymbol{b}}: x \neq p\right\} \cong \mathbb{R}^{2}$.

Under the inclusion map $\iota_{\boldsymbol{a}}: H_{*}\left(S_{\boldsymbol{a}} \cap S_{\boldsymbol{b}}\right) \hookrightarrow H_{*}\left(S_{\boldsymbol{a}}\right)$ we have $\iota_{\boldsymbol{a}}\left(c_{1}\right)=c(\varnothing)$ and $\iota_{\boldsymbol{a}}\left(c_{2}\right)=c(\{(1,2)\})+c(\{(3,4)\})$. Similarly under the inclusion $\iota_{\boldsymbol{b}}: H_{*}\left(S_{\boldsymbol{a}} \cap S_{\boldsymbol{b}}\right) \hookrightarrow$ $H_{*}\left(S_{\boldsymbol{b}}\right)$ we have $\iota_{\boldsymbol{b}}\left(c_{1}\right)=c(\varnothing)$ and $\iota_{\boldsymbol{b}}\left(c_{2}\right)=c(\{(1,4)\})+c(\{(2,3)\})$. This proves that $\psi^{-}\left(H_{*}\left(S_{\boldsymbol{a}} \cap S_{\boldsymbol{b}}\right)\right)$ is generated by Type I and Type II relations whenever $\boldsymbol{a}$ and $\boldsymbol{b}$ differ on two arcs. 
Consider $\boldsymbol{a}, \boldsymbol{b} \in B^{3,1}$ where $\boldsymbol{a}$ has ray (1) and arc (2,3) while $\boldsymbol{b}$ has ray (3) and arc $(1,2)$. In this case $\boldsymbol{a} w(\boldsymbol{b})$ consists of one line and no circles, so $S_{\boldsymbol{a}} \cap S_{\boldsymbol{b}}$ is just a point. Call this point $c_{1}$. Under the inclusion map $\iota_{\boldsymbol{a}}: H_{*}\left(S_{\boldsymbol{a}} \cap S_{\boldsymbol{b}}\right) \hookrightarrow H_{*}\left(S_{\boldsymbol{a}}\right)$ we have $\iota_{\boldsymbol{a}}\left(c_{1}\right)=c(\varnothing)$. Under the inclusion $\iota_{\boldsymbol{b}}: H_{*}\left(S_{\boldsymbol{a}} \cap S_{\boldsymbol{b}}\right) \hookrightarrow H_{*}\left(S_{\boldsymbol{b}}\right)$ we get $\iota_{\boldsymbol{b}}\left(c_{1}\right)=c(\varnothing)$. This proves that whenever $\boldsymbol{a}$ and $\boldsymbol{b}$ differ on an arc and a ray, the image $\psi^{-}\left(H_{*}\left(S_{\boldsymbol{a}} \cap S_{\boldsymbol{b}}\right)\right)$ is generated by Type III relations.

Definition 4.4. A standard dotted noncrossing matching is a dotted noncrossing matching where no dotted arc is nested beneath any other arc and no ray is to the right of any dotted arc. Figure 10 has an example.

Lemma 4.5. The set of standard noncrossing matchings contains a set of generators for the collection of complex vector spaces $H_{*}\left(X_{n-k, k}\right)$.

Proof. Type I and Type II relations allow us to reduce to a set of generators where no dotted arcs are nested beneath undotted or dotted arcs. Type III relations allow us to move rays to the left of all dotted arcs.

Young tableaux theory is often used to study irreducible representations of the symmetric group. Recall that a Young diagram corresponding to a partition of the number $n$ is a collection of top and left aligned boxes with row lengths given by the partition. A standard Young tableau is a filling of a Young diagram with the numbers 1 through $n$ so that numbers strictly increase from left to right and top to bottom. For more information on Young tableaux and the Specht module theory we will use in the next section we refer the reader to [Fulton 1997, Chapter 7].

Results from [De Concini and Procesi 1981, p. 213] and [Garsia and Procesi 1992, Equation 4.2], specialized to the two-row case, yield the following statement, which gives us the dimension of $H_{*}\left(X_{n-k, k}\right)$ in each degree.

Proposition 4.6. For each $m \leq k$ the Springer action on $H_{2 m}\left(X_{n-k, k}\right)$ is isomorphic to the irreducible representation of the symmetric group $S_{n}$ corresponding to the partition $(n-m, m)$.

Classical results about representations of the symmetric group together with Proposition 4.6 tell us that the dimension of $H_{2 m}\left(X_{n-k, k}\right)$ is therefore the number of standard fillings of a Young diagram of shape $(n-m, m)$. As we show in the following lemma, it turns out that this dimension is also equal to the number of standard noncrossing matchings of type $(n-k, k)$ with $m$ undotted arcs.

Lemma 4.7. Standard noncrossing matchings of type $(n-k, k)$ with $m$ undotted arcs are in bijection with standard Young tableaux of shape $(n-m, m)$.

Proof. We give an explicit bijection between standard dotted noncrossing matchings and standard Young tableaux.

Let $M$ be a standard dotted noncrossing matching. Define $g(M)$ to be the tableau obtained by writing the right hand endpoint of each undotted arc of $M$ on the 
bottom row and all other numbers on the top. The tableau $g(M)$ is standard since for each number in the bottom row, the left endpoint of that arc is written in the top row. The tableau has shape $(n-m, m)$ where $m$ is the number of undotted arcs in $M$.

Now let $T$ be a standard tableau of shape $(n-m, m)$. We define $h(T)$ to be the standard dotted noncrossing matching with $n-2 k$ rays and $m$ undotted arcs constructed as follows. Start with the smallest number in the bottom row, draw an undotted arc with right endpoint incident on that number and left endpoint incident on the nearest unoccupied vertex. Repeat this for all remaining numbers in the bottom row. Then in the leftmost $n-2 k$ empty positions place $n-2 k$ rays. Fill the remaining empty positions with unnested dotted arcs. Figure 10 has an example of this map.

\begin{tabular}{|l|l|l|l|l|}
\hline 1 & 2 & 4 & 5 & 7 \\
\hline 3 & 6 & &
\end{tabular}

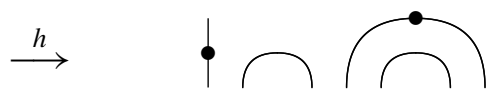

Figure 10. A standard tableau of shape $(5,2)$ and its associated standard dotted noncrossing matching of type $(4,3)$.

The proof that $g \circ h$ and $h \circ g$ produce the identity on the sets of standard tableaux and standard dotted noncrossing matchings respectively is a straightforward generalization of the one given in [Russell and Tymoczko 2008, Section 2.2].

Corollary 4.8. The standard noncrossing matchings on $n$ vertices with $n-2 k$ rays and $m$ undotted arcs form a basis for the complex vector space $H_{2 m}\left(X_{n-k, k}\right)$. In particular, when $m=k$ there are no dotted arcs and we recover the bijection from [Stroppel and Webster 2010, Proposition 3].

Proof. By Proposition 4.6 the dimension of $H_{2 m}\left(X_{n-k, k}\right)$ is the same as the dimension of the irreducible representation of $S_{n}$ corresponding to the partition ( $n-$ $m, m)$. Classical representation theory tells us that this dimension is equal to the number of standard Young tableaux of shape $(n-m, m)$ [Fulton 1997].

By Lemma 4.5 the set of standard dotted noncrossing matchings with $m$ undotted arcs is a generating set for $H_{2 m}\left(X_{n-k, k}\right)$. Lemma 4.7 tells us that this generating set has the proper dimension and therefore is a basis.

\section{Springer action on $H_{*}\left(X_{n-k, k}\right)$}

Consider $n$ and $0 \leq k \leq\lfloor n / 2\rfloor$. In [Russell and Tymoczko 2008] we define a graded action of the symmetric group $S_{2 n-2 k}$ on $H_{*}\left(X_{n-k, n-k}\right)$. We proved that this action which can be combinatorially and diagrammatically described is isomorphic to the Springer action. In other words we prove that the action of $S_{2 n-2 k}$ that we define 


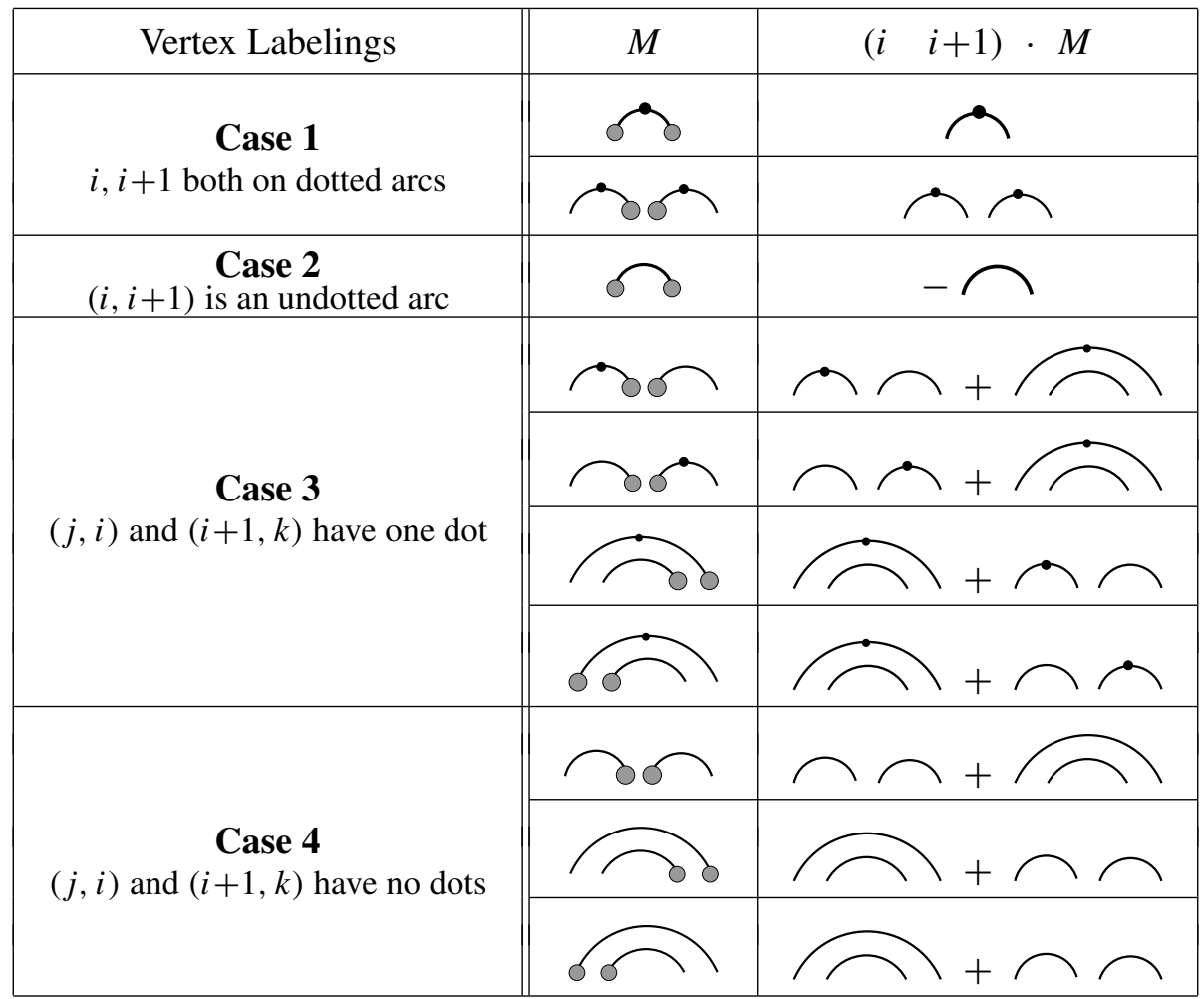

Figure 11. The $S_{n}$-action on standard dotted noncrossing matchings. (Gray vertices indicate positions $i$ and $i+1$.)

on $H_{2 m}\left(X_{n-k, n-k}\right)$ is the irreducible representation of $S_{2 n-2 k}$ corresponding to the partition $(2 n-2 k-m, m)$ for each $0 \leq m \leq n-k$. This action is summarized by the chart in Figure 11.

Using the same approach as [Russell and Tymoczko 2008, Section 4] we construct a symmetric group action on the homology of all two-row Springer varieties that is isomorphic to the Springer action. We once again describe this action diagrammatically. Finally we give a convenient skein-theoretic method for calculating the action.

Defining a symmetric group action. Define the map $\eta: X_{n-k, k} \rightarrow X_{n-k, n-k}$ to be

$$
\eta\left(x_{1}, \ldots, x_{n}\right)=\left( \pm p, \mp p, \ldots, p,-p, x_{1}, \ldots, x_{n}\right) .
$$

Recall that the completion map $\varphi$ from Section 3 builds a matching of type ( $n-$ $k, n-k)$ from one of type $(n-k, k)$ by anchoring rays to the left of the matching. Given some noncrossing matching $\boldsymbol{a} \in B^{n-k, k}$ it is an immediate consequence of the 
definition of $\eta$ that $\eta\left(S_{\boldsymbol{a}}\right) \subset S_{\varphi(\boldsymbol{a})}$. In other words $\eta$ maps the component associated to $\boldsymbol{a}$ into the component associated to its completion.

Recall the antipodal map $\gamma: X_{n-k, k} \rightarrow\left(S^{2}\right)^{n}$ defined in the proof of Theorem 2.7:

$$
\gamma\left(x_{1}, \ldots, x_{n}\right)=\left(-x_{1}, x_{2}, \ldots,(-1)^{n} x_{n}\right) .
$$

Finally consider the map $\iota_{n}:\left(S^{2}\right)^{n} \rightarrow\left(S^{2}\right)^{2 n-2 k}$ given by

$$
\iota_{n}\left(x_{1}, \ldots, x_{n}\right)=\left((-1)^{n-1} p, \ldots,(-1)^{n-1} p,(-1)^{n} x_{1}, \ldots,(-1)^{n} x_{n}\right) .
$$

Lemma 5.1. The following diagram is commutative.

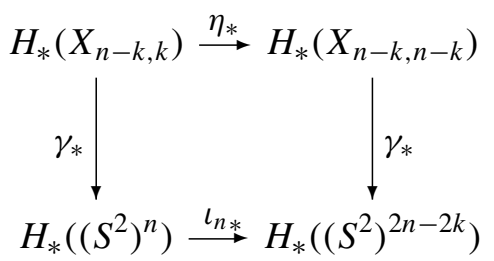

Proof. On one hand we have

$$
\begin{aligned}
\iota_{n} \circ \gamma\left(x_{1}, \ldots, x_{n}\right)= & \iota_{n}\left(-x_{1}, x_{2}, \ldots,(-1)^{n-1} x_{n-1},(-1)^{n} x_{n}\right) \\
= & \left((-1)^{n-1} p, \ldots,(-1)^{n-1} p,\right. \\
& \left.\quad(-1)^{n+1} x_{1},(-1)^{n+2} x_{2}, \ldots,(-1)^{2 n-1} x_{n-1},(-1)^{2 n} x_{n}\right) .
\end{aligned}
$$

On the other hand,

$$
\begin{aligned}
\gamma \circ \eta\left(x_{1}, \ldots, x_{n}\right) & =\gamma\left((-1)^{n-2 k} p,(-1)^{n-2 k-1} p, \ldots,(-1)^{2} p,(-1)^{1} p, x_{1}, \ldots, x_{n}\right) \\
& =\left((-1)^{n-2 k+1} p, \ldots,(-1)^{n-2 k+1} p,\right. \\
& \left.(-1)^{n-2 k+1} x_{1}, \ldots,(-1)^{2 n-2 k-1} x_{n-1},(-1)^{2 n-2 k} x_{n}\right) .
\end{aligned}
$$

The parity of $n$ and $n-2 k$ must be the same. Therefore $(-1)^{n-2 k+i}=(-1)^{n+i}$ and the diagram commutes.

Proposition 5.2. The map $\gamma_{*}: H_{*}\left(X_{n-k, k}\right) \rightarrow H_{*}\left(\left(S^{2}\right)^{n}\right)$ is injective, and the action of $S_{n}$ on $H_{*}\left(\left(S^{2}\right)^{n}\right)$ given by permutation of coordinates stabilizes the image of $H_{*}\left(X_{n-k, k}\right)$ under $\gamma_{*}$.

Proof. Viewing the maps $\eta$ and $\iota_{n}$ on diagrammatic homology generators it is straightforward to see that $\eta_{*}$ and $\iota_{n *}$ are injective. In [Russell and Tymoczko 2008, Corollary 3.13] we prove that $\gamma_{*}: H_{*}\left(X_{n-k, n-k}\right) \rightarrow H_{*}\left(\left(S^{2}\right)^{2 n-2 k}\right)$ is injective. Therefore by Lemma 5.1 we see that $\gamma_{*}: H_{*}\left(X_{n-k, k}\right) \rightarrow H_{*}\left(\left(S^{2}\right)^{n}\right)$ is injective and the first half of the lemma is proven.

Consider the subgroup $G \leq S_{2 n-2 k}$ fixing the numbers $1, \ldots, n-2 k$. Then $G$ is isomorphic to $S_{n}$ via the map

$$
(i \quad j) \mapsto(i+n-2 k \quad j+n-2 k) .
$$


The action of $G$ on $H_{*}\left(X_{n-k, n-k}\right)$ is given by pulling back the action of $G$ on $H_{*}\left(\left(S^{2}\right)^{2 n-2 k}\right)$. Since the homology of a cartesian product of copies of $S^{2}$ is a tensor product of copies of $H_{*}\left(S^{2}\right)$, the symmetric group action that permutes tensor factors commutes with the inclusion map $\iota_{n *}$. We can therefore prove the second part of the lemma by showing that the action of $G$ on $H_{*}\left(X_{n-k, n-k}\right)$ stabilizes the image of $H_{*}\left(X_{n-k, k}\right)$ under $\eta_{*}$.

Again note that given $\boldsymbol{a} \in B^{n-k, k}$ we have $\eta\left(S_{\boldsymbol{a}}\right) \subset S_{\varphi(\boldsymbol{a})}$. On the level of homology $\eta$ sends generators in $H_{*}\left(X_{n-k, k}\right)$ to their associated completions in $H_{*}\left(X_{n-k, n-k}\right)$. The new dotted arcs in the completed generators will have right endpoint at the vertex where the ray was formerly incident and left endpoint on one of the first $n-2 k$ vertices. While this new dotted noncrossing matching is not necessarily standard, it is equivalent to a unique standard noncrossing matching via Type II relations.

Diagrammatically, the image $\eta_{*}\left(H_{*}\left(X_{n-k, k}\right)\right)$ consists of exactly those generators in $H_{*}\left(X_{n-k, n-k}\right)$ with no undotted arcs incident on the first $n-2 k$ vertices. Indeed the preimage of such a generator under $\eta_{*}$ is the standard dotted noncrossing matching with the first $n-2 k$ vertices removed, identical undotted arcs, and the unique standard arrangement of rays and dotted arcs in the remaining positions.

In order to prove that the subgroup action of $G$ stabilizes $\eta_{*}\left(H_{*}\left(X_{n-k, k}\right)\right)$ we need to show that each simple transposition $(i \quad i+1)$, where $n-2 k+1 \leq i<$ $2 n-2 k$, maps elements of $\eta_{*}\left(H_{*}\left(X_{n-k, k}\right)\right)$ to a linear combination of elements of $H_{*}\left(X_{n-k, n-k}\right)$ with no undotted arcs incident on the first $n-2 k$ vertices. Examining rows 1, 2, 4, and 7 on the chart in Figure 11 we see that it is not possible for such a simple transposition to map a generator without an undotted arc incident on the first $n-2 k$ vertices to one with an undotted arc incident on the first $n-2 k$ vertices.

Theorem 5.3. There is a graded $S_{n}$-action on $H_{*}\left(X_{n-k, k}\right)$ described by the chart in Figure 11 together with the chart in Figure 12.

Proof. Using the commutative diagram in Lemma 5.1 together with the chart in Figure 11 we are able to calculate $\gamma_{*}^{-1}\left((i \quad i+1) \cdot \gamma_{*}(M)\right)$ where $M \in H_{*}\left(X_{n-k, k}\right)$. By Proposition 5.2 we have a well-defined $S_{n}$-action given by

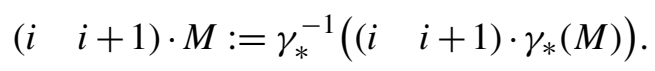

Verifying the Springer action. Young tabloids are equivalence classes of Young tableaux where the equivalence relation is permutation of numbers within rows. The symmetric group $S_{n}$ acts on tabloids; indeed given $\sigma \in S_{n}$ and a tabloid $T$ we let $\sigma \cdot T$ be the tabloid with $\sigma(i)$ replacing $i$ for all $i=1, \ldots, n$. Consider the vector space $U_{\lambda}$ of complex linear combinations of tabloids of shape $\lambda$. For a 


\begin{tabular}{|c|c|c|}
\hline Vertex Labelings & $M$ & $(i \quad i+1) \cdot M$ \\
\hline $\begin{array}{c}\text { Case } 5 \\
i, i+1 \text { both on rays }\end{array}$ & $\$$ & $\phi \phi$ \\
\hline $\begin{array}{c}\text { Case } 6 \\
i, i+1 \text { on ray and dotted arc }\end{array}$ & & \\
\hline \multirow{2}{*}{$\begin{array}{c}\text { Case } 7 \\
i, i+1 \text { on ray and undotted arc }\end{array}$} & & 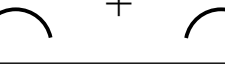 \\
\hline & & 1 \\
\hline
\end{tabular}

Figure 12. The $S_{n}$-action on matchings of type $(n-k, k)$ with rays. (Gray vertices indicate positions $i$ and $i+1$.)

tabloid $T$ write its corresponding element in $U_{\lambda}$ as $v_{T}$. Then the action of $S_{n}$ on tabloids induces an action on $U_{\lambda}$.

Consider $0 \leq m \leq\lfloor n / 2\rfloor$ and $\lambda=(n-m, m)$ a partition of the number $n$. For each tableau $T$ let $\operatorname{Col}(T)$ be the subgroup of permutations in $S_{n}$ that stabilize all columns of $T$. For each tableau $T$ define the following vector in $U_{n-m, m}$.

$$
e_{T}=\sum_{\sigma \in \operatorname{Col}(T)} \operatorname{sign}(\sigma) v_{\sigma \cdot T}
$$

The subspace of $U_{n-m, m}$ generated by these vectors which we denote $V_{n-m, m}$ is known as the Specht module. A classical result says that the symmetric group action on $V_{n-m, m}$ given by permuting entries in tabloids is the irreducible representation of $S_{n}$ corresponding to the partition $(n-m, m)$. Furthermore $V_{n-m, m}$ has a basis given by $\left\{e_{T}\right\}$ where $T$ are standard tableaux.

Given a standard dotted noncrossing matching $M$ of type $(n-k, k)$ with $m$ undotted $\operatorname{arcs}$ let $\operatorname{Undot}(M)$ be the subgroup of $S_{n}$ generated by all transpositions $(i \quad j)$ where $(i, j)$ is an undotted arc in $M$. Let $e_{M}$ be the vector in $U_{n-m, m}$ defined as

$$
e_{M}=\sum_{\sigma \in \operatorname{Undot}(T)} \operatorname{sign}(\sigma) v_{\sigma \cdot h(M)} .
$$

Definition 5.4. Let the subspace of $U_{n-m, m}$ generated by the vectors $e_{M}$ the matching module. Denote the matching module by $W_{n-m, m}$.

Define a map

$$
\zeta: H_{2 m}\left(X_{n-k, k}\right) \rightarrow U_{n-m, m}
$$

by $\zeta(M)=e_{M}$. Then the image of $\zeta$ is exactly the matching module.

Define the map

$$
f: U_{n-m, m} \rightarrow U_{2 n-2 k-m, m}
$$




$$
T=\begin{array}{|l|l|l|l|l|}
\hline 1 & 4 & 5 & 6 & 7
\end{array} \quad T^{\prime}=\begin{array}{|l|l|l|l|l|l|l|}
\hline 1 & 2 & 5 & 6 & 7 & 8 \\
\hline 2 & 3 & & 4
\end{array}
$$

Figure 13. The behavior of tabloids under the map $f$ where $n=7$ and $k=3$.

as $f\left(v_{T}\right)=v_{T^{\prime}}$, where $T^{\prime}$ is the tabloid with numbers 1 through $n-2 k$ in the top row and $i+n-2 k$ in the row where $i$ appears in $T$. Note that $f$ is injective. Figure 13 has an example.

Lemma 5.5. Let $M$ be a standard dotted noncrossing matching. Then $f\left(e_{M}\right)=$ $e_{\varphi(M)}$ where $\varphi(M)$ is understood to be the completion of the associated matching along with dotting information.

Proof. Recall that $h$ is the bijection taking standard Young tableaux to standard dotted noncrossing matchings. If $(i, j)$ is an undotted arc in $M$ then $(i+n-$ $2 k, j+n-2 k)$ is an undotted arc in $\varphi(M)$. This means that $h(M)^{\prime}=h(\varphi(M))$. Furthermore if $\operatorname{Undot}(M)$ is generated by transpositions $(i \quad j)$ then $\operatorname{Undot}(\varphi(M))$ is generated by transpositions $(i+n-2 k \quad j+n-2 k)$.

The bijection $(i \quad j) \mapsto(i+n-2 k \quad j+n-2 k)$ between $S_{n}$ and $G$ restricts to a bijection between $\operatorname{Undot}(M)$ and $\operatorname{Undot}(\varphi(M))$. Moreover for $\sigma \in \operatorname{Undot}(M)$ and its corresponding $\sigma^{\prime} \in \operatorname{Undot} \varphi(M)$ we have $(\sigma \cdot \theta(M))^{\prime}=\sigma^{\prime} \cdot \theta(\varphi(M))$. Thus we conclude that $f\left(e_{M}\right)=e_{\varphi(M)}$.

Corollary 5.6. The following diagram is commutative:

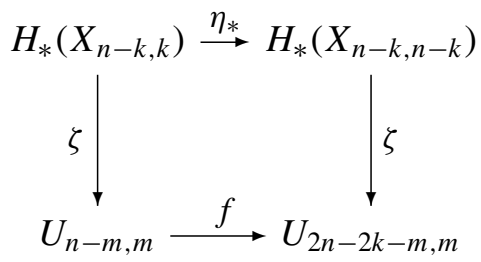

Lemma 5.7. The action of $S_{n}$ on $U_{n-m, m}$ commutes with that of $G$ on $U_{2 n-2 k-m, m}$.

Proof. Let $\sigma^{\prime}$ be the element of $G$ corresponding to $\sigma \in S_{n}$ via the bijection described above. For $v_{T} \in U_{n-m, m}$ we want to show that $f\left(\sigma \cdot v_{T}\right)=\sigma^{\prime} \cdot f\left(v_{T}\right)=v_{\sigma^{\prime} \cdot T^{\prime}}$.

The tabloid $\sigma \cdot T$ has $\sigma(i)$ in the row that $i$ occupied in $T$. The tabloid corresponding to $f\left(\sigma \cdot v_{T}\right)$ has $1, \ldots, n-2 k$ in the top row and $\sigma(i)+n-2 k$ in the row that $i$ occupied in $T$. On the other hand $T^{\prime}$ has $1, \ldots, n-2 k$ in the top row and $i+n-2 k$ in the row that $i$ occupied in $T$. The tabloid $\sigma^{\prime} \cdot T^{\prime}$ has $1, \ldots, n-2 k$ on the top row and $\sigma^{\prime}(i+n-2 k)$ in the row that $i$ occupied in $T$. Since $\sigma^{\prime}(i+n-2 k)=\sigma(i)+n-2 k$ we conclude that $f\left(\sigma \cdot v_{T}\right)=\sigma^{\prime} \cdot f\left(v_{T}\right)$.

Corollary 5.8. The map $\zeta: H_{2 m}\left(X_{n-k, k}\right) \rightarrow W_{n-m, m}$ is an $S_{n}$-equivariant isomorphism of complex vector spaces. 
Proof. As we noted earlier $\eta_{*}$ is injective, so $\eta_{*}: H_{2 m}\left(X_{n-k, k}\right) \rightarrow \eta_{*}\left(H_{2 m}\left(X_{n-k, k}\right)\right.$ is an isomorphism. In [Russell and Tymoczko 2008, Lemma 4.2] we prove that

$$
\zeta: H_{2 m}\left(X_{n-k, n-k}\right) \rightarrow W_{2 n-2 k-m, m} \subset U_{2 n-2 k-m, m}
$$

is an $S_{2 n-2 k}$-equivariant isomorphism, so it follows that

$$
\zeta: \eta_{*}\left(H_{2 m}\left(X_{n-k, k}\right)\right) \rightarrow f\left(W_{n-m, m}\right)
$$

is a $G$-equivariant isomorphism. Finally Lemma 5.5 implies that $f$ is injective, so $f: W_{n-m, m} \rightarrow f\left(W_{n-m, m}\right)$ is an isomorphism. Thus

$$
\zeta=f^{-1} \circ \zeta \circ \eta_{*}: H_{2 m}\left(X_{n-k, k}\right) \rightarrow W_{n-m, m}
$$

is an isomorphism.

By construction the action of $S_{n}$ on $H_{2 m}\left(X_{n-k, k}\right)$ commutes with the action of $G$ on $\eta_{*}\left(H_{2 m}\left(X_{n-k, k}\right)\right)$. Since $\zeta: H_{2 m}\left(X_{n-k, n-k}\right) \rightarrow U_{2 n-2 k-m, m}$ is $S_{2 n-2 k-}$ equivariant it is also $G$-equivariant. This information together with Lemma 5.7 proves that $\zeta: H_{2 m}\left(X_{n-k, k}\right) \rightarrow W_{n-m, m}$ is $S_{n}$-equivariant.

Theorem 5.9. The Specht module $V_{n-m, m}$ and the matching module $W_{n-m, m}$ are equal as $S_{n}$-representations. Thus the $S_{n}$ action on $H_{*}\left(X_{n-k, k}\right)$ is isomorphic to the Springer representation.

Proof. Since $V_{n-m, m}$ and $W_{n-m, m}$ are subspaces of $U_{n-m, m}$ of the same dimension each equipped with an $S_{n}$-action, the intersection $V_{n-m, m} \cap W_{n-m, m}$ is also an $S_{n}$ representation. The Specht module $V_{n-m, m}$ is irreducible, so $V_{n-m, m} \cap W_{n-m, m}$ is either 0 or $V_{n-m, m}$.

Let $M$ be the standard dotted noncrossing matching with $m$ undotted arcs $(1,2)$, $(3,4), \ldots,(2 m-1,2 m)$. Then $M$ represents a basis element in $H_{2 m}\left(X_{n-k, k}\right)$. Furthermore the columns of $h(M)$ are exactly the undotted $\operatorname{arcs}$ of $M$, so $\operatorname{Undot}(M)=$ $\operatorname{Col}(h(M))$. This means that $e_{M}=e_{h(M)}$, so the matching module and Specht module always have a common vector.

Since $V_{n-m, m} \cap W_{n-m, m}$ is nonempty we conclude that the intersection is all of $V_{n-m, m}$. We therefore conclude that $V_{n-m, m}=W_{n-m, m}$. By the equivariance proved in Corollary 5.8 the $S_{n}$ action we have defined on $H_{*}\left(X_{n-k, k}\right)$ is isomorphic to the unique irreducible representation of $S_{n}$ corresponding to the partition $(n-m, m)$. Therefore we conclude that the action we have defined on the homology of the Springer variety is isomorphic to the Springer action.

A skein-theoretic formulation of the Springer action. The Springer action on $H_{*}\left(X_{n-k, k}\right)$ is described diagrammatically in the charts in Figures 11 and 12 . We can also describe the action skein-theoretically. 
Theorem 5.10. Given standard dotted noncrossing matching $M \in H_{*}\left(X_{n-k, k}\right)$ and $\sigma \in S_{n}$ glue a flattened braid corresponding to $\sigma$ to the bottom of $M$ forming $M^{\prime}$. Then the Springer action $\sigma \cdot M$ is equal to $s\left(M^{\prime}\right)$, where $s$ is defined as follows.

$$
\begin{aligned}
& s(\searrow)=s\left(\begin{array}{l}
\vdots \\
\vdots
\end{array}\right)+s(\vdots) \\
& s\left(M^{\prime} \sqcup \bigcirc\right)=s\left(-2 M^{\prime}\right) \quad s\left(M^{\prime} \sqcup \bigcirc\right)=0
\end{aligned}
$$

As an example, consider the permutation ( $\left(\begin{array}{lll}1 & 2 & 3\end{array}\right)$ acting on the following generator of $H_{2}\left(X_{2,1}\right)$.

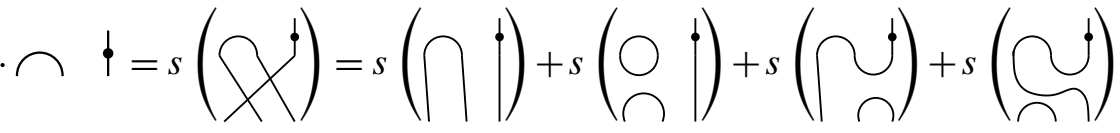

$$
\begin{aligned}
& =\cap \phi-2 \cap \phi+\phi \cap+\cap \phi=\phi \cap
\end{aligned}
$$

\section{Appendix}

In Section 3 we cited [Khovanov 2004] as the source for Proposition 3.9 and the base case of Lemma 3.14. Proofs of these results were left to the reader in that paper. For the sake of completeness we include the proofs here. Throughout the appendix we take $n=2 m$.

Proof of Proposition 3.9. Let $|\boldsymbol{a} w(\boldsymbol{b})|$ be denoted by $c$. This proof proceeds by induction on $c$. We begin with the case that $c=1$ and then argue by induction. The following algorithm finds a sequence of $m-1$ moves on $\boldsymbol{a}$ that yields $\boldsymbol{b}$. An arc $(i, j)$ in $\boldsymbol{b}$ is said to be unpaired in $\boldsymbol{a} w(\boldsymbol{b})$ if $\boldsymbol{a}$ does not have $\operatorname{arc}(i, j)$. Let $\boldsymbol{a}=\boldsymbol{a}_{0}$ and let $t=0$.

(1) If $\boldsymbol{b}$ has no unpaired $\operatorname{arcs}$ in $\boldsymbol{a}_{t} w(\boldsymbol{b})$, then $\boldsymbol{a}_{t}=\boldsymbol{b}$ and we are done. Otherwise begin with the narrowest leftmost arc $(i, j)$ in $\boldsymbol{b}$ that is unpaired in $\boldsymbol{a}_{t} w(\boldsymbol{b})$. Then $\boldsymbol{a}_{t}$ has $\operatorname{arcs}(i, k),(j, l)$ where $i, j, k, l$ have no particular order.

(2) Perform $\rightarrow$ or $\leftarrow$ in $\boldsymbol{a}_{t}$ to produce a new matching $\boldsymbol{a}_{t+1}$ with $\operatorname{arcs}(i, j)$ and $(k, l)$.

(3) Increment $t$ by 1 , and repeat the first two steps.

It is always possible to perform $\rightarrow$ or $\leftarrow$ as required. At each step the algorithm guarantees all arcs nested beneath $(i, j)$ are paired in $\boldsymbol{a}_{t} w(\boldsymbol{b})$ since arcs beneath $(i, j)$ are narrower than $(i, j)$. Thus each arc in $\boldsymbol{a}_{t}$ either has zero or two endpoints between vertices $i$ and $j$. This means that the $\operatorname{arcs}(i, k)$ and $(j, l)$ are adjacent in $\boldsymbol{a}_{t}$, and $\rightarrow$ or $\leftarrow$ can be performed to get a new matching $\boldsymbol{a}_{t+1}$ with arcs $(i, j)$ and $(k, l)$. 
The number of circles in $\boldsymbol{a}_{t+1} w(\boldsymbol{b})$ is one more than the number of circles in $\boldsymbol{a}_{t} w(\boldsymbol{b})$. To see this note that the $\operatorname{arcs}(i, k),(j, l)$ in $\boldsymbol{a}_{t}$ and the $\operatorname{arc}(i, j)$ in $\boldsymbol{b}$ are all part of the same circle in $\boldsymbol{a}_{t} w(\boldsymbol{b})$. The move that produces $\boldsymbol{a}_{t+1}$ must change the number of circles. Since this move acts on two arcs that are part of a single circle, the only possibility is that the move increases the number of circles by one.

Since we increase the number of circles with each iteration of our algorithm, this process terminates after $m-1$ iterations at which point we will have $m$ circles - the maximum number of circles possible. This proves that $d(\boldsymbol{a}, \boldsymbol{b}) \leq m-1$.

In an arbitrary sequence of moves, each time $\boldsymbol{a}_{t} \rightarrow \boldsymbol{a}_{t+1}$ or $\boldsymbol{a}_{t} \leftarrow \boldsymbol{a}_{t+1}$ is performed the number of circles in $\boldsymbol{a}_{t} w(\boldsymbol{b})$ is either one greater or one less than $\boldsymbol{a}_{t+1} w(\boldsymbol{b})$ depending on whether the move taking $\boldsymbol{a}_{t}$ to $\boldsymbol{a}_{t+1}$ joins two circles or splits a single circle. Since we start with one circle, it will require at least $m-1$ moves to produce $m$ circles. This means our algorithm generates a minimal sequence $\left(\boldsymbol{a}=\boldsymbol{a}_{0}, \boldsymbol{a}_{1}, \ldots, \boldsymbol{a}_{m-2}, \boldsymbol{a}_{m-1}=\boldsymbol{b}\right)$ and $d(\boldsymbol{a}, \boldsymbol{b})=m-1$.

Now consider $\boldsymbol{a}, \boldsymbol{b} \in B^{m, m}$ where $\boldsymbol{a} w(\boldsymbol{b})$ has $c$ circles, and enumerate the circles $\alpha_{1}, \ldots, \alpha_{c}$. Each circle $\alpha_{r}$ passes through the horizontal axis $2 q_{r}$ times. By the argument above, we have

$$
d(\boldsymbol{a}, \boldsymbol{b})=\sum_{1 \leq r \leq c}\left(q_{r}-1\right)=\left(\sum_{1 \leq r \leq c} q_{r}\right)-c=m-c .
$$

Proof of the base case for Lemma 3.14. This is proven using induction on the distance between $\boldsymbol{a}$ and $\boldsymbol{b}$. If there exists a minimal sequence of moves taking $\boldsymbol{a}$ to $\boldsymbol{b}$ that begins with $\leftarrow$ the inductive step is clear. The lemmas below are required in order to handle the case that no minimal sequences of moves taking $\boldsymbol{a}$ to $\boldsymbol{b}$ begin with $\leftarrow$. When this occurs, the following arguments prove that there always exists a minimal sequence for $\boldsymbol{a}$ and $\boldsymbol{b}$ of the form $(\rightarrow, \rightarrow, \ldots, \rightarrow, \rightarrow)$. In other words, we want to show that in this case we take $\boldsymbol{a}=\boldsymbol{c}$.

Lemma 5.11. Let $\boldsymbol{a}$ be the matching with arcs

$$
(1,2),(3,4), \ldots,(n-3, n-2),(n-1, n)
$$

and $\boldsymbol{b}$ the matching with arcs

$$
(2,3),(4,5), \ldots,(n-4, n-3),(n-2, n-1),(1, n) .
$$

Then there exists a minimal sequence $\left(\boldsymbol{a} \rightarrow \boldsymbol{a}_{1} \ldots \boldsymbol{a}_{m-1} \rightarrow \boldsymbol{b}\right)$.

Proof. Consider the minimal sequence generated by the algorithm in the proof of Proposition 3.9. Each move takes the matching with arcs $(1, i),(i+1, i+2)$ to the matching with arcs $(1, i+2),(i, i+1)$. Since $\operatorname{arcs}(1, i),(i+1, i+2)$ are unnested these moves always have the form $\rightarrow$. 
This next lemma addresses circles of this type within more complicated gluings of two matchings.

Lemma 5.12. Let $i_{1}<\cdots<i_{p}$. Let $\boldsymbol{a}$ be some matching with arcs $\left(i_{1}, i_{2}\right), \ldots$, $\left(i_{p-1}, i_{p}\right)$. Let $\boldsymbol{b}$ be some matching with arcs $\left(i_{2}, i_{3}\right), \ldots,\left(i_{p-2}, i_{p-1}\right),\left(i_{1}, i_{p}\right)$. There exists a minimal sequence for $\boldsymbol{a}$ and $\boldsymbol{b}$ where all moves on arcs incident on the vertices $i_{1}, \ldots, i_{p}$ have the form $\rightarrow$.

Proof. We prove this using induction. Assume $i_{1}<i_{2}<i_{3}<i_{4}$ and that $\boldsymbol{a}$ is a matching with arcs $\left(i_{1}, i_{2}\right),\left(i_{3}, i_{4}\right)$ while $\boldsymbol{b}$ is a matching with $\operatorname{arcs}\left(i_{2}, i_{3}\right),\left(i_{1}, i_{4}\right)$. The algorithm given in the proof of Proposition 3.9 will eventually find arc $\left(i_{2}, i_{3}\right)$ unpaired in $\boldsymbol{a}_{t} w(\boldsymbol{b})$. It will perform the move $\rightarrow$ taking the unnested pair of arcs $\left(i_{1}, i_{2}\right),\left(i_{3}, i_{4}\right)$ to the nested pair of arcs $\left(i_{2}, i_{3}\right),\left(i_{1}, i_{4}\right)$.

Assume that the result is true for some $p$. Consider $i_{1}<\cdots<i_{p+2}$. Take $a$ and $\boldsymbol{b}$ to be matchings as in the statement of the corollary. The algorithm from the proof of Proposition 3.9 will eventually find the narrowest arc in $\boldsymbol{b}$ incident on these vertices. Say this arc is $\left(i_{k}, i_{k+1}\right)$. The move $\rightarrow$ will be performed on matching $\boldsymbol{a}_{t}$ producing new $\operatorname{arcs}\left(i_{k-1}, i_{k+2}\right),\left(i_{k}, i_{k+1}\right)$. The arc $\left(i_{k}, i_{k+1}\right)$ is now paired in $\boldsymbol{a}_{t+1} w(\boldsymbol{b})$. After renumbering the remaining arcs are incident on $p$ vertices as in the statement of the corollary. We apply the inductive hypothesis to the remaining arcs, and the result follows by induction.

Before the next lemma, we provide one technical definition that will make the statement of the lemma easier.

Definition 5.13. Let $i<j<k<l$ and suppose $(i, l),(j, k)$ are nested arcs in some matching $\boldsymbol{a}$. We say that an $\operatorname{arc}$ is between $(i, l)$ and $(j, k)$ if the arc in $\boldsymbol{a}$ with left endpoint between $i$ and $j$ and right endpoint between $k$ and $l$.

Lemma 5.14. Let $\boldsymbol{a}, \boldsymbol{b} \in B^{m, m}$. Let $i<j<k<l$ and suppose $(i, l),(j, k)$ are nested arcs in $\boldsymbol{a}$ that are part of the same circle in $\boldsymbol{a} w(\boldsymbol{b})$ such that there is no arc in $\boldsymbol{a}$ lying between $(i, l)$ and $(j, k)$ that is also part of that circle. Then the number of arcs between $(i, l),(j, k)$ is even.

Proof. Given some circle $c$ in $\boldsymbol{a} w(\boldsymbol{b})$ let $W(c, z)$ be the winding number of $c$ about $z$. Consider a line segment between $z_{0}, z_{1} \notin c$ which is transverse to $c$. Say that the number of intersections of that segment with the circle $c$ is some number $r$. Then mod 2 intersection theory tells us that

$$
W\left(c, z_{0}\right) \equiv W\left(c, z_{1}\right)+r(\bmod 2) .
$$

Take some arc between $(i, l)$ and $(j, k)$. Say this arc is part of circle $c$ in $\boldsymbol{a} w(\boldsymbol{b})$. Note that the circle $c$ is not the same circle that $(i, l)$ and $(j, k)$ are part of. Vertices $i$ and $j$ are both either inside or outside the circle $c$. Consider the vertices $i$ and $j$ to be points in the plane. Then $W(i, c) \equiv W(j, c)(\bmod 2)$. Putting this together 
with the previous statement, say the segment between $i$ and $j$ intersects $c$ exactly $r$ times. Then $W(i, c) \equiv W(j, c)+r(\bmod 2)$ and hence $r \equiv 0(\bmod 2)$. We conclude that $c$ has an even number of intersections with the line segment between $i$ and $j$.

Each arc in $c$ with both endpoints between $i$ and $j$ contributes an even number of intersections, so the number of arcs between $(i, l)$ and $(j, k)$ must also be even. Since this is true for each circle with some arc between $(i, l)$ and $(j, k)$, there is an even number of arcs between $(i, l)$ and $(j, k)$.

Lemma 5.15. Within the collection of arcs between $(i, l)$ and $(j, k)$ there are two adjacent arcs that are part of the same circle.

Proof. By the argument above, there is an even number of vertices between $i$ and $j$. Given two circles $c$ and $c^{\prime}$ with arcs that lie between $(i, l)$ and $(k, j)$ the argument in the previous proof says that there are an even number of arcs from $c^{\prime}$ between any pair of arcs in $c$.

Because there are finitely many vertices between $i$ and $j$, there must be some pair of arcs from a single circle that have no arcs from any other circle between them. Otherwise we could find infinitely many arcs between $(i, l)$ and $(j, k)$.

Lemma 5.16. Given $\boldsymbol{a}, \boldsymbol{b} \in B^{m, m}$ if every minimal sequence $\left(\boldsymbol{a}, \boldsymbol{a}_{1}, \ldots, \boldsymbol{a}_{m-1}, \boldsymbol{b}\right)$ begins with $\boldsymbol{a} \rightarrow \boldsymbol{a}_{1}$ then there exists a minimal sequence $(\rightarrow, \rightarrow, \cdots, \rightarrow, \rightarrow)$.

Proof. Assume that all minimal sequences for $\boldsymbol{a}$ and $\boldsymbol{b}$ begin with $\rightarrow$. In particular this means that there does not exist a pair of nested arcs in $\boldsymbol{a}$ that are part of the same circle in $\boldsymbol{a} w(\boldsymbol{b})$ and have no other arc from that same circle between them. (The existence of such a pair would violate our assumption that all minimal sequences begin with $\rightarrow$.)

Say there is some pair of nested $\operatorname{arcs}(i, l),(j, k) \in \boldsymbol{a}$ that are part of the same circle in $\boldsymbol{a} w(\boldsymbol{b})$. By Lemma 5.15 there must be a pair of nested, adjacent arcs in $\boldsymbol{a}$ contributed by some other circle of $\boldsymbol{a} w(\boldsymbol{b})$ lying between $(i, l)$ and $(j, k)$. In this case, we could perform $\leftarrow$ on this pair once again violating our assumption that all minimal sequences must begin with $\rightarrow$. We conclude that the no pair of nested arcs in $\boldsymbol{a}$ are part of the same circle in $\boldsymbol{a} w(\boldsymbol{b})$.

Since we have just shown the arcs in each circle in $\boldsymbol{a} w(\boldsymbol{b})$ are pairwise unnested, they have the form

$$
\left(i_{1}, i_{2}\right),\left(i_{3}, i_{4}\right), \ldots,\left(i_{t-1}, i_{t}\right) \text { where } i_{1}<i_{2}<\cdots<i_{t-1}<i_{t} .
$$

The arcs in $\boldsymbol{b}$ that comprise the rest of this circle necessarily have the form

$$
\left(i_{2}, i_{3}\right), \ldots,\left(i_{t-2}, i_{t-1}\right),\left(i_{1}, i_{t}\right) .
$$

Consider the minimal sequence of moves for $\boldsymbol{a}$ and $\boldsymbol{b}$ obtained by the algorithm in the proof of Proposition 3.9. By Lemma 5.12 each move has the form $\rightarrow$. 
Therefore we have a minimal sequence consisting only of $\rightarrow$ moves, and the lemma is proven.

Having established Lemma 5.16 we can now easily finish the proof of the base case for Lemma 3.14.

We proceed using induction. If the distance between matchings is 1 , the result is clear since there is only one move between matchings in any minimal sequence.

Now assume the lemma is true for all $\boldsymbol{a}, \boldsymbol{b}$ with $d(\boldsymbol{a}, \boldsymbol{b}) \leq m-1$. Assume that $d(\boldsymbol{a}, \boldsymbol{b})=m$. Let $\left(\boldsymbol{a}=\boldsymbol{a}_{0}, \boldsymbol{a}_{1}, \ldots, \boldsymbol{a}_{m-1}, \boldsymbol{a}_{m}=\boldsymbol{b}\right)$ be a minimal sequence of moves from $\boldsymbol{a}$ to $\boldsymbol{b}$ such that the move $\leftarrow$ occurs as early as possible.

If $\boldsymbol{a}_{0} \leftarrow \boldsymbol{a}_{1}$ we can apply the inductive assumption to get $\boldsymbol{a}_{1} \succ \boldsymbol{c} \prec \boldsymbol{a}_{m}$ with $d\left(\boldsymbol{a}_{1}, \boldsymbol{a}_{m}\right)=d\left(\boldsymbol{a}_{1}, \boldsymbol{c}\right)+d\left(\boldsymbol{c}, \boldsymbol{a}_{m}\right)$ and $\left(\boldsymbol{a}_{1} \leftarrow \cdots \leftarrow \boldsymbol{c} \rightarrow \cdots \rightarrow \boldsymbol{a}_{m}\right)$. This proves the lemma in this case since we have a minimal sequence of the form

$$
\left(\boldsymbol{a} \leftarrow \boldsymbol{a}_{1} \leftarrow \cdots \leftarrow \boldsymbol{c} \rightarrow \cdots \rightarrow \boldsymbol{b}\right) .
$$

If $\boldsymbol{a}_{0} \rightarrow \boldsymbol{a}_{1}$ then there does not exist a minimal sequence for $\boldsymbol{a}$ and $\boldsymbol{b}$ with first move $\leftarrow$. By Lemma 5.16 there exists in this case a minimal sequence of the form $(\boldsymbol{a} \rightarrow \cdots \rightarrow \boldsymbol{b})$.

\section{Acknowledgements}

Many thanks to Charlie Frohman and Julianna Tymoczko for their continued support and guidance as well as many helpful conversations related to this work.

\section{References}

[Borho and MacPherson 1981] W. Borho and R. MacPherson, "Représentations des groupes de Weyl et homologie d'intersection pour les variétés nilpotentes", C. R. Acad. Sci. Paris Sér. I Math. 292:15 (1981), 707-710. MR 82f:14002 Zbl 0467.20036

[Cautis and Kamnitzer 2008] S. Cautis and J. Kamnitzer, "Knot homology via derived categories of coherent sheaves. I. The $\mathfrak{s l}(2)$-case”, Duke Math. J. 142:3 (2008), 511-588. MR 2009i:57025

[De Concini and Procesi 1981] C. De Concini and C. Procesi, "Symmetric functions, conjugacy classes and the flag variety", Invent. Math. 64:2 (1981), 203-219. MR 82m:14030 Zbl 0475.14041

[Fulton 1997] W. Fulton, Young tableaux: with applications to representation theory and geometry, London Mathematical Society Student Texts 35, Cambridge University Press, 1997. MR 99f:05119 Zbl 0878.14034

[Fung 2003] F. Y. C. Fung, "On the topology of components of some Springer fibers and their relation to Kazhdan-Lusztig theory", Adv. Math. 178:2 (2003), 244-276. MR 2004m:20087 Zbl 1035. 20004

[Garsia and Procesi 1992] A. M. Garsia and C. Procesi, "On certain graded $S_{n}$-modules and the q-Kostka polynomials”, Adv. Math. 94:1 (1992), 82-138. MR 93j:20030 Zbl 0797.20012

[Hotta 1981] R. Hotta, “On Springer's representations”, J. Fac. Sci. Univ. Tokyo Sect. IA Math. 28:3 (1981), 863-876. MR 83h:20038 Zbl 0584.20033 
[Kazhdan and Lusztig 1980] D. Kazhdan and G. Lusztig, “A topological approach to Springer's representations”, Adv. in Math. 38:2 (1980), 222-228. MR 82f:20076 Zbl 0458.20035

[Khovanov 2002] M. Khovanov, "A functor-valued invariant of tangles", Algebr. Geom. Topol. 2 (2002), 665-741. MR 2004d:57016 Zbl 1002.57006

[Khovanov 2004] M. Khovanov, "Crossingless matchings and the cohomology of $(n, n)$ Springer varieties", Commun. Contemp. Math. 6:4 (2004), 561-577. MR 2005g:14090 Zbl 1079.57009

[Lusztig 1981] G. Lusztig, "Green polynomials and singularities of unipotent classes", Adv. in Math. 42:2 (1981), 169-178. MR 83c:20059 Zbl 0473.20029

[Russell 2009] H. M. Russell, "The Bar-Natan skein module of the solid torus and the homology of $(n, n)$ Springer varieties”, Geom. Dedicata 142 (2009), 71-89. MR 2011b:57014 Zbl 1176.57018

[Russell and Tymoczko 2008] H. Russell and J. Tymoczko, "Springer representations on the Khovanov Springer fibers", preprint, 2008. arXiv 0811.0650v1

[Seidel and Smith 2006] P. Seidel and I. Smith, "A link invariant from the symplectic geometry of nilpotent slices”, Duke Math. J. 134:3 (2006), 453-514. MR 2007f:53118 Zbl 1108.57011

[Slodowy 1980] P. Slodowy, Four lectures on simple groups and singularities, Communications of the Mathematical Institute, Rijksuniversiteit Utrecht 11, Rijksuniversiteit Utrecht Mathematical Institute, Utrecht, 1980. MR 82b:14002 Zbl 0425.22020

[Spaltenstein 1976] N. Spaltenstein, "The fixed point set of a unipotent transformation on the flag manifold”, Nederl. Akad. Wetensch. Proc. Ser. A 79 = Indag. Math. 38:5 (1976), 452-456. MR 58 \#5700

[Springer 1976] T. A. Springer, "Trigonometric sums, Green functions of finite groups and representations of Weyl groups”, Invent. Math. 36 (1976), 173-207. MR 56 \#491 Zbl 0374.20054

[Stroppel 2005] C. Stroppel, "Categorification of the Temperley-Lieb category, tangles, and cobordisms via projective functors", Duke Math. J. 126:3 (2005), 547-596. MR 2005i:17011 Zbl 1112. 17010

[Stroppel 2009] C. Stroppel, "Parabolic category O, perverse sheaves on Grassmannians, Springer fibres and Khovanov homology", Compos. Math. 145:4 (2009), 954-992. MR 2011a:17014 Zbl 1187.17004

[Stroppel and Webster 2010] C. Stroppel and B. Webster, "2-block Springer fibers: convolution algebras, coherent sheaves, and embedded TQFT", (2010). To appear in Comm. Math. Helvetici. arXiv 0802.1943

[Vargas 1979] J. A. Vargas, "Fixed points under the action of unipotent elements of $\mathrm{SL}_{n}$ in the flag variety”, Bol. Soc. Mat. Mexicana (2) 24:1 (1979), 1-14. MR 81h:14027 Zbl 0458.14019

[Wehrli 2009] S. Wehrli, "A remark on the topology of $(n, n)$ Springer varieties", preprint, 2009. arXiv 0908.2185

Received July 9, 2010. Revised October 26, 2010.

HEATHER M. RuSSELL

DEPARTMENT OF MATHEMATICS

UNIVERSITY OF SOUTHERN CALIFORNIA

3620 South Vermont AVEnue, KAP 108

LOS ANGELES, CA 90089-2532

UNITED STATES

heathemr@usc.edu 


\title{
PACIFIC JOURNAL OF MATHEMATICS
}

\author{
http://www.pjmath.org \\ Founded in 1951 by
}

E. F. Beckenbach (1906-1982) and F. Wolf (1904-1989)

\section{EDITORS}

V. S. Varadarajan (Managing Editor)

Department of Mathematics

University of California

Los Angeles, CA 90095-1555

pacific@math.ucla.edu

Vyjayanthi Chari

Department of Mathematics

University of California

Riverside, CA 92521-0135

chari@math.ucr.edu

\section{Robert Finn}

Department of Mathematics Stanford University

Stanford, CA 94305-2125

finn@math.stanford.edu

Kefeng Liu

Department of Mathematics

University of California

Los Angeles, CA 90095-1555

liu@math.ucla.edu
Darren Long

Department of Mathematics

University of California

Santa Barbara, CA 93106-3080

long@math.ucsb.edu

Jiang-Hua Lu

Department of Mathematics

The University of Hong Kong

Pokfulam Rd., Hong Kong jhlu@maths.hku.hk

Alexander Merkurjev

Department of Mathematics University of California

Los Angeles, CA 90095-1555 merkurev@math.ucla.edu
Sorin Popa

Department of Mathematics

University of California

Los Angeles, CA 90095-1555

popa@math.ucla.edu

Jie Qing

Department of Mathematics

University of California

Santa Cruz, CA 95064

qing@ cats.ucsc.edu

Jonathan Rogawski

Department of Mathematics

University of California

Los Angeles, CA 90095-1555

jonr@math.ucla.edu

\section{PRODUCTION}

pacific@math.berkeley.edu

Silvio Levy, Scientific Editor Matthew Cargo, Senior Production Editor

ACADEMIA SINICA, TAIPEI

CALIFORNIA INST. OF TECHNOLOGY

INST. DE MATEMÁTICA PURA E APLICADA

KEIO UNIVERSITY

MATH. SCIENCES RESEARCH INSTITUTE

NEW MEXICO STATE UNIV.

OREGON STATE UNIV.

\section{SUPPORTING INSTITUTIONS}

STANFORD UNIVERSITY
UNIV. OF BRITISH COLUMBIA
UNIV. OF CALIFORNIA, BERKELEY
UNIV. OF CALIFORNIA, DAVIS
UNIV. OF CALIFORNIA, LOS ANGELES
UNIV. OF CALIFORNIA, RIVERSIDE
UNIV. OF CALIFORNIA, SAN DIEGO
UNIV. OF CALIF., SANTA BARBARA

UNIV. OF CALIF., SANTA CRUZ

UNIV. OF MONTANA

UNIV. OF OREGON

UNIV. OF SOUTHERN CALIFORNIA

UNIV. OF UTAH

UNIV. OF WASHINGTON

WASHINGTON STATE UNIVERSITY

These supporting institutions contribute to the cost of publication of this Journal, but they are not owners or publishers and have no responsibility for its contents or policies.

See inside back cover or www.pjmath.org for submission instructions.

The subscription price for 2011 is US \$420/year for the electronic version, and \$485/year for print and electronic.

Subscriptions, requests for back issues from the last three years and changes of subscribers address should be sent to Pacific Journal of Mathematics, P.O. Box 4163, Berkeley, CA 94704-0163, U.S.A. Prior back issues are obtainable from Periodicals Service Company, 11 Main Street, Germantown, NY 12526-5635. The Pacific Journal of Mathematics is indexed by Mathematical Reviews, Zentralblatt MATH, PASCAL CNRS Index, Referativnyi Zhurnal, Current Mathematical Publications and the Science Citation Index.

The Pacific Journal of Mathematics (ISSN 0030-8730) at the University of California, c/o Department of Mathematics, 969 Evans Hall, Berkeley, CA 94720-3840, is published monthly except July and August. Periodical rate postage paid at Berkeley, CA 94704, and additional mailing offices. POSTMASTER: send address changes to Pacific Journal of Mathematics, P.O. Box 4163, Berkeley, CA 94704-0163.

PJM peer review and production are managed by EditFLOW ${ }^{\mathrm{TM}}$ from Mathematical Sciences Publishers.

PUBLISHED BY PACIFIC JOURNAL OF MATHEMATICS

at the University of California, Berkeley 94720-3840

A NON-PROFIT CORPORATION

Typeset in IATEX

Copyright $(2011$ by Pacific Journal of Mathematics 


\title{
PACIFIC JOURNAL OF MATHEMATICS
}

\author{
Volume $253 \quad$ No. $1 \quad$ September 2011
}

Singularities of the projective dual variety

ROLAND ABUAF

Eigenvalue estimates for hypersurfaces in $\mathbb{H}^{m} \times \mathbb{R}$ and applications

Pierre BÉRARD, Philippe CASTILlon and Marcos CaVAlCante

Conformal Invariants associated to a measure: Conformally covariant operators

Sun-Yung A. Chang, Matthew J. Gursky and Paul Yang

Compact symmetric spaces, triangular factorization, and Cayley coordinates

DEREK HABERMAS

Automorphisms of the three-torus preserving a genus-three Heegaard splitting

JESSE JOHNSON

The rationality problem for purely monomial group actions

HiDETAKA KiTAYAMA

On a Neumann problem with $p$-Laplacian and noncoercive resonant nonlinearity

Salvatore A. Marano and Nikolaos S. Papageorgiou

Minimal ramification in nilpotent extensions

NADYA MARKIN and STEPHEN V. ULLOM

Regularity of weakly harmonic maps from a Finsler surface into an $n$-sphere

XIAOHUAN MO and LIANG ZHAO

On the sum of powered distances to certain sets of points on the circle

NikOlai NikOlOV and RAFAEL RAFAilov

Formal geometric quantization II

PAUL-ÉMILE PARADAN

Embedded constant-curvature curves on convex surfaces

HAROLD ROSENBERG and MATTHIAS SCHNEIDER

A topological construction for all two-row Springer varieties 